\title{
NUMERICAL SOLUTION OF AN EVOLUTION EQUATION WITH A POSITIVE-TYPE MEMORY TERM
}

\author{
W. McLEAN ${ }^{1}$ and V. THOMÉE ${ }^{2}$
}

(Received 2 December 1990; revised 4 February 1992)

\begin{abstract}
We study the numerical solution of an initial-boundary value problem for a Volterra type integro-differential equation, in which the integral operator is a convolution product of a positive-definite kernel and an elliptic partial-differential operator. The equation is discretised in space by the Galerkin finite-element method and in time by finite differences in combination with various quadrature rules which preserve the positive character of the memory term. Special attention is paid to the case of a weakly singular kernel. Error estimates are derived and numerical experiments reported.
\end{abstract}

\section{Introduction}

We shall consider initial-boundary value problems of the form

$$
\begin{aligned}
u_{t}(t)+\int_{0}^{t} \beta(t-s) A u(s) d s & =f(t), & & \text { in } \Omega, \text { for } t \geq 0 . \\
u & =0, & & \text { on } \partial \Omega, \text { for } t>0, \\
u(0) & =v, & & \text { in } \Omega .
\end{aligned}
$$

Here $u_{t}=\partial u / \partial t, A$ is a second-order self-adjoint positive-definite elliptic differential operator, and $\beta$ is a positive-definite kernel, i.e., $\beta \in L_{1, l o c}\left(\overline{\mathbf{R}}_{+}\right)$,

\footnotetext{
'School of Mathematics, University of New South Wales, Sydney 2033

${ }^{2}$ Department of Mathematics, Chalmers University of Technology, S-412 96 Göteborg, Sweden

(C) Australian Mathematical Society, 1993, Serial-fee code 0334-2700/93
} 
where $\mathbf{R}_{+}=(0, \infty)$, and satisfies

$$
\int_{0}^{T} \int_{0}^{t} \beta(t-s) \varphi(s) d s \varphi(t) d t \geq 0, \quad \forall T>0, \quad \varphi \in C([0, T]) .
$$

We assume that all functions occurring are real-valued.

Equations of the above type, and particularly nonlinear versions thereof, are used to model phenomena in viscoelasticity and heat conduction in materials with memory, e.g. MacCamy [12, 13] and Jin Choi and MacCamy [2]. Their mathematical properties have also been studied in MacCamy and Wong [14], Londen [10], Dafermos and Nohel [5] and Staffans [18]. For $\beta$ smooth on $\overline{\mathbf{R}}_{+}$ they are hyperbolic in character whereas if $\beta$ has a weak singularity at 0 , such as if $\beta(t)=t^{\alpha-1} / \Gamma(\alpha), 0<\alpha<1$, then they adopt a parabolic behaviour, more so the stronger the singularity. This latter kernel is of particular interest, cf. Jin Choi and MacCamy [2].

We recall that $\beta$ is positive definite if and only if

$$
\operatorname{Re} \hat{\beta}(i \theta)=\int_{0}^{\infty} \beta(t) \cos (\theta t) d t \geq 0, \quad \forall \theta \in \mathbf{R},
$$

where $\hat{\beta}$ denotes the Laplace transform of $\beta$, and that a sufficient condition for this to hold is that $\beta \in L_{1}\left(\mathbf{R}_{+}\right) \cap C^{2}\left(\mathbf{R}_{+}\right)$and $(-1)^{j} \beta^{(j)} \geq 0$ on $\mathbf{R}_{+}$for $j=0,1,2$. This class of functions contains the totally positive functions, i.e., functions which may be represented as

$$
\beta(t)=\int_{0}^{\infty} e^{-s t} d \mu(s),
$$

where $\mu$ is a positive measure with the appropriate number of finite moments.

The hypothesis (1.2) on $\beta$ easily implies the stability property

$$
\|u(T)\| \leq\|v\|+2 \int_{0}^{T}\|f(t)\| d t, \quad \text { for } T>0, \text { where }\|\cdot\|=\|\cdot\|_{L_{2}(\Omega)} .
$$

To show this, simply take inner products in $L_{2}(\Omega)$ of both sides of (1.1) with $2 u(t)$, and then integrate over $[0, T]$ to obtain

$$
\|u(T)\|^{2}+2 \int_{0}^{T} \int_{0}^{t} \beta(t-s) A(u(s), u(t)) d s d t \leq\|v\|^{2}+2 \int_{0}^{T}\|f(t)\|\|u(t)\| d t,
$$

for each $T \geq 0$, where $A(\cdot, \cdot)$ is the bilinear form associated with the elliptic operator $A$. It is easy to see that (1.2) implies that the double integral is nonnegative (cf. Lemma 2.1 below), and (1.4) then easily follows. 
We shall study the numerical solution of (1.1) and consider first discretisation in space by a Galerkin finite-element method. Let thus $\left\{S_{h}\right\}$ be a family of finite-dimensional subspaces of $H_{0}^{1}(\Omega)$ with the approximation property

$$
\inf _{\chi \in S_{h}}\left\{\|v-\chi\|+h\|v-\chi\|_{1}\right\} \leq C h^{r}\|v\|_{r}, \quad \text { for } v \in H_{0}^{1}(\Omega) \cap H^{r}(\Omega),
$$

where $\|\cdot\|_{r}$ denotes the norm in $H^{r}(\Omega)$. In applications, $h$ is typically the maximum diameter of a triangle in the triangulation underlying the definition of the finite element space $S_{h}$ (cf., e.g., Ciarlet [3]). We define the spatially semidiscrete problem by

$$
\begin{aligned}
\left(u_{h, l}, \chi\right)+\int_{0}^{t} \beta(t-s) A\left(u_{h}(s), \chi\right) d s & =(f, \chi), \quad \forall \chi \in S_{h}, t \geq 0, \\
u_{h}(0) & =v_{h},
\end{aligned}
$$

where $(\cdot, \cdot)$ is the inner product in $L_{2}(\Omega)$. Setting $\chi=u_{h}(t)$ and integrating in time we find easily that this semidiscrete problem inherits the stability property (1.4) of the continuous problem. As a result of this, we show in a routine manner an error estimate of the form

$$
\left\|u_{h}(t)-u(t)\right\| \leq\left\|v_{h}-v\right\|+C(u) h^{r}, \quad \text { for } t \geq 0,
$$

as well as an optimal-order error estimate for $\nabla u_{h}$.

We then turn to discretisation of (1.1) in time. We introduce a time step $k$, set $t_{n}=n k$, and let $U^{n}$ be the approximation of $u_{n}=u\left(t_{n}\right)$. A natural approach for time stepping is to replace $u_{t}$ by a difference quotient such as the backward difference quotient

$$
\bar{\partial}_{t} U^{n}=\frac{U^{n}-U^{n-1}}{k},
$$

and then replace the integral in (1.1) by a finite sum so that the equations become

$$
\begin{aligned}
\left(\bar{\partial}_{t} U^{n}, \chi\right)+\sum_{j=0}^{n} \omega_{n j} A\left(U^{j}, \chi\right) & =\left(f_{n}, \chi\right), \quad \text { for } n \geq 1, \forall \chi \in S_{h}, \\
U^{0} & =v_{h},
\end{aligned}
$$

with $f_{n}=f\left(t_{n}\right)$. Setting

$$
q_{n}(\Phi)=\sum_{j=0}^{n} \omega_{n j} \Phi^{j},
$$


it is then natural to try to choose the quadrature coefficients in such a manner that the following analogue of (1.2) holds, namely

$$
Q_{N}(\Phi)=k \sum_{n=1}^{N} q_{n}(\Phi) \Phi^{n} \geq 0, \quad \forall N \geq 1, \quad \Phi=\left(\Phi^{0}, \ldots, \Phi^{N}\right)^{\top}
$$

We shall call such a quadrature formula positive. We note that this is not possible, in general, unless the $\omega_{n 0}$ vanish. In our technical work we shall therefore have to introduce some modifications of this concept. In the particular case that $q_{n}(\varphi)$ is of convolution type, so that $\omega_{n j}=b_{n-j}$, for $1 \leq j \leq n$, the positivity of $q_{n}(\varphi)$ is related to a discrete analogue of (1.3), namely

$$
\sum_{j=0}^{\infty} b_{j} \cos j \theta \geq 0, \quad \forall \theta \in \mathbf{R} .
$$

One natural choice of quadrature formula is the rectangle rule determined by the values of the integrand at the right-hand end-points of the time intervals, so that $\omega_{n j}=k \beta_{n-j}$ for $0<j \leq n$, where $\beta_{j}=\beta\left(t_{j}\right)$, with $\omega_{n 0}=0$. As we shall see, this choice does satisfy (1.8), and we may then derive an error estimate of the form

$$
\left\|U^{n}-u\left(t_{n}\right)\right\| \leq\left\|v_{h}-v\right\|+C(u)\left(h^{r}+k\right) .
$$

Another, perhaps equally natural, choice would be to use a rectangle rule based on the left-hand end-points of the intervals, i.e., to choose $\omega_{n n}=0$ and $\omega_{n j}=k \beta_{n-j}$ for $j<n$. This would have the added advantage of making the equation explicit, or at least only dependent on the mass matrix, at each time level. It turns out, however, that the property (1.8) does not hold for this choice. Thus, just as in the case of the standard heat equation, the implicit method has better stability properties than the explicit one.

The quadrature rules just described are first-order accurate, thus matching the first-order accuracy of the backward time difference quotient. One could also choose the second-order accurate trapezoidal rule, which turns out to be a positive quadrature formula, and combine this with the second-order accurate, three-level backward difference operator $D_{t}^{(2)} U^{n}=\bar{\partial}_{t} U^{n}+\frac{1}{2} k \bar{\partial}_{t}^{2} U^{n}$. This method is stable, and we may show an error estimate that is $O\left(h^{r}+k^{2}\right)$.

As in the Crank-Nicolson scheme for the heat equation, it would also be natural to consider $\bar{\partial}_{t} U^{n}$ as a second-order accurate approximation to $u_{t}\left(t_{n-1 / 2}\right)$, and then choose $q_{n}$ to approximate the integral with upper limit $t=t_{n-1 / 2}$. Doing this by an average between the trapezoidal approximations at $t=t_{n}$ and 
$t_{n-1}$ does not produce a positive quadrature formula in the above sense, but nevertheless yields a stable scheme of order $O\left(h^{r}+k^{2}\right)$ as can be seen by a slight modification of the arguments.

One of the difficulties in a numerical method such as (1.6) is that if $\omega_{n j} \neq 0$ for $j \leq n$, then all values of the solution $U^{j}, j=1, \ldots, n$, have to be retained, causing great demands for storage of the data. This is in contrast to the situation for a parabolic or hyperbolic differential equation, where only a fixed low number of time levels is involved at each time step, and the data can be discarded as the computation goes along. As a way around this difficulty in the case of a parabolic integro-differential equation (with a term of the form $A u$ included on the left in the equation) it was proposed in Sloan and Thomee [17] that the quadrature be based on fewer points, thus reducing the number of time levels at which the data need to be saved. Unfortunately, it does not seem to be possible to combine this approach with the positivity of the quadrature rule. One case which appears to be common in practice, and when this problem can be handled, is when $\beta(t)$ is a linear combination of a small number of exponential functions.

Earlier work on the numerical solution of problems of type (1.1) has been done by e.g. Neta [15], Jin Choi and MacCamy [2], Sanz-Serna [16], and LopezMarcos [11]. We shall make some comments on these papers below. Fairweather [7] considers continuous in time and backward Euler-type time-stepping methods based on spline collocation in space; see also Yan and Fairweather [21]. The use of fast transform methods for the time discretisation has been studied by Yan [20].

The rest of the paper is organised as follows. In Section 2 we treat the spatially semidiscrete problem. Section 3 is concerned with the discretisation in time. Here we introduce various positivity concepts for the quadrature rules used to approximate the integral in (1.1), and show corresponding stability results. Further, these stability results are used to obtain preliminary error bounds for the completely discrete schemes, containing one term which depends on the as-yetunspecified quadrature rule. In Section 4 we study specific quadrature schemes and relate them to the results in Section 3. In Section 5 we discuss the regularity of the solution of (1.1) with particular reference to the regularity requirements of our error estimates. It is shown that for $\beta$ smooth, all estimates needed can be shown under appropriate assumptions on the data. For the weakly-singular kernel $\beta(t)=t^{\alpha-1} / \Gamma(\alpha)$, however, it turns out that, even with smooth data, $u_{t t}(t)=O\left(t^{\alpha-1}\right)$ and $u_{t t t}(t)=O\left(t^{\alpha-2}\right)$ for small $t$, so that $u_{t t}$ is integrable at $t=0$, but $u_{t t}$ is not. This will suffice for optimal-order convergence for a first-order, but not for a second-order, method. Finally, Section 6 describes some 
simple numerical experiments, the results of which agree with our theoretical analysis.

\section{Discretisation in space}

In this section we shall consider the discretisation in space of the initialboundary value problem (1.1). The numerical solution is sought, for each $t \geq 0$, in a finite-dimensional space $S_{h} \subset H_{0}^{1}(\Omega)$, depending on a small parameter $h$, which we assume to have the property that (1.5) holds, uniformly in $h$.

Writing (1.1) in weak form as

$$
\begin{aligned}
\left(u_{t}, \varphi\right)+\int_{0}^{t} \beta(t-s) A(u(s), \varphi) d s & =(f, \varphi), \quad \forall \varphi \in H_{0}^{1}(\Omega), t \geq 0, \\
u(0) & =v,
\end{aligned}
$$

we define the semidiscrete solution $u_{h}:[0, \infty) \rightarrow S_{h}$ by

$$
\begin{aligned}
\left(u_{h, t}, \chi\right)+\int_{0}^{t} \beta(t-s) A\left(u_{h}(s), \chi\right) d s & =(f, \chi), \quad \forall \chi \in S_{h}, t \geq 0(2.1) \\
u_{h}(\cdot, 0) & =v_{h},
\end{aligned}
$$

where $v_{h}$ is an appropriate approximation of $v$ in $S_{h}$. It is easy to see that this latter finite-dimensional problem has a unique solution.

We shall first show an $L_{2}$-error estimate for (2.1). For this purpose we need the following lemma.

LEMMA 2.1. Let $B$ be a positive definite selfadjoint operator in a Hilbert space, with $B(\cdot, \cdot)$ the corresponding symmetric bilinear form on $D\left(B^{1 / 2}\right)$, and let $\beta$ be a positive definite kernel. Then

$$
\begin{aligned}
& B_{T}(u)=\int_{0}^{T} \int_{0}^{t} \beta(t-s) B((s), u(t)) d s d t \geq 0, \\
& \forall T>0, u \in C\left([0, T] ; D\left(B^{1 / 2}\right)\right) .
\end{aligned}
$$

PROOF. Letting $\left\{\lambda_{j}\right\}_{j=1}^{\infty}$ and $\left\{\varphi_{j}\right\}_{j=1}^{\infty}$ be the eigenvalues and eigenfunctions of $B$, and letting $u_{j}=\left(u, \varphi_{j}\right)$, we have

$$
B_{T}(u)=\sum_{j=1}^{\infty} \lambda_{j} \int_{0}^{T} \int_{0}^{t} \beta(t-s) u_{j}(s) u_{j}(t) d s d t .
$$


Since each of these integrals is positive by the positive definiteness of the kernel $\beta$, the result follows.

THEOREM 2.1. Assume that $\beta$ is a positive definite kernel, and that $\left\{S_{h}\right\}$ satisfies (1.5). Then under the appropriate regularity assumptions we have, for the solutions of (2.1) and (1.1),

$$
\left\|u_{h}(t)-u(t)\right\| \leq\left\|v_{h}-v\right\|+C h^{r}\left\{\|v\|_{r}+\int_{0}^{t}\left\|u_{t}\right\|_{r} d s\right\}, \quad \text { for } t \geq 0 .
$$

PROOF. As is usual for parabolic and hyperbolic equations, we introduce the Ritz projection $R_{h}: H_{0}^{1}(\Omega) \rightarrow S_{h}$ by

$$
A\left(R_{h} u-u, \chi\right)=0, \quad \forall \chi \in S_{h},
$$

and write the error

$$
u_{h}-u=\left(u_{h}-R_{h} u\right)+\left(R_{h} u-u\right)=\theta+\rho .
$$

From a well-known error estimate for the elliptic problem [3], we have at once

$$
\|\rho(t)\|+h\|\rho(t)\|_{1} \leq C h^{r}\|u(t)\|_{r} \leq C h^{r}\left\{\|v\|_{r}+\int_{0}^{t}\left\|u_{t}\right\|_{r} d s\right\},
$$

and it remains to bound $\theta(t)$. We have by our definitions

$$
\begin{aligned}
\left(\theta_{t}, \chi\right)+ & \int_{0}^{t} \beta(t-s) A(\theta(s), \chi) d s \\
& =(f, \chi)-\left(R_{h} u_{t}, \chi\right)-\int_{0}^{t} \beta(t-s) A\left(R_{h} u_{t}(s), \chi\right) d s \\
& =-\left(\rho_{t}, \chi\right), \quad \forall \chi \in S_{h}, t \geq 0,
\end{aligned}
$$

where in the last step we have used the definition (2.3) in the integral term. Since $\theta \in S_{h}$ we may choose $\chi=\theta$ to obtain

$$
\frac{1}{2} \frac{d}{d t}\|\theta(t)\|^{2}+\int_{0}^{t} \beta(t-s) A(\theta(s), \theta(t)) d s=-\left(\rho_{t}(t), \theta(t)\right) .
$$

By integration over $(0, T)$ this yields

$\frac{1}{2}\left(\|\theta(T)\|^{2}-\|\theta(0)\|^{2}\right)+\int_{0}^{T} \int_{0}^{t} \beta(t-s) A(\theta(s), \theta(t)) d s d t \leq \int_{0}^{T}\left\|\rho_{t}\right\|\|\theta\| d t$. 
Lemma 2.1 shows that the double integral is nonnegative, and hence

$$
\|\theta(T)\|^{2} \leq\|\theta(0)\|^{2}+2 \int_{0}^{T}\left\|\rho_{t}\right\|\|\theta\| d t, \quad \text { for } T \geq 0 .
$$

For a given $T$, letting $t_{0}$ be such that $\left\|\theta\left(t_{0}\right)\right\|=\sup _{0 \leq t \leq T}\|\theta(t)\|$, we conclude from (2.6) that

$$
\|\theta(T)\|^{2} \leq\left\|\theta\left(t_{0}\right)\right\|^{2} \leq\left\{\|\theta(0)\|+2 \int_{0}^{t_{0}}\left\|\rho_{t}\right\| d t\right\}\left\|\theta\left(t_{0}\right)\right\|,
$$

and so

$$
\|\theta(T)\| \leq\|\theta(0)\|+2 \int_{0}^{T}\left\|\rho_{t}\right\| d t .
$$

Here,

$$
\|\theta(0)\| \leq\left\|v_{h}-v\right\|+\left\|R_{h} v-v\right\| \leq\left\|v_{h}-v\right\|+C h^{r}\|v\|_{r},
$$

and applying (2.4) to $u_{t}$ we conclude that $\left\|\rho_{t}\right\| \leq C h^{r}\left\|u_{t}\right\|$, and so

$$
\|\theta(T)\| \leq\left\|v_{h}-v\right\|+C h^{r}\left\{\|v\|_{r}+\int_{0}^{T}\left\|u_{t}\right\|_{r} d t\right\} .
$$

Together (2.4) and (2.7) complete the proof of (2.2).

We now turn to an error estimate in $H^{1}(\Omega)$.

THEOREM 2.2. Assume that $\beta$ is a positive-definite kernel, and that the finiteelement spaces $\left\{S_{h}\right\}$ satisfy (1.5) and are such that the orthogonal projection $P_{h}$ of $L_{2}(\Omega)$ onto $S_{h}$ is bounded in $H^{1}(\Omega)$, uniformly in $h$. Then under the appropriate regularity assumptions we have, for the solutions of (2.1) and (1.1),

$$
\left\|u_{h}(t)-u(t)\right\|_{1} \leq C\left\|v_{h}-v\right\|_{1}+C h^{r-1}\left\{\|v\|_{r}+\int_{0}^{t}\left\|u_{t}\right\|_{r} d s\right\}, \quad \text { for } t \geq 0 .
$$

PROOF. Since the $H^{1}$-norm of $\rho=R_{h} u-u$ is bounded by the right-hand side of (2.8), it suffices to estimate $\theta=u_{h}-R_{h} u$. Let $A_{h}: S_{h} \rightarrow S_{h}$ be the discrete analogue of $A$ defined by

$$
\left(A_{h} \psi, \chi\right)=A(\psi, \chi), \quad \forall \psi, \chi \in S_{h} .
$$


Then, setting $\chi=A_{h} \theta$ in (2.5) we have $A\left(\theta_{t}, \theta\right)+\int_{0}^{t} \beta(t-s)\left(A_{h} \theta(s), A_{h} \theta(t)\right) d s=-\left(\rho_{t}, A_{h} \theta(t)\right)=-A\left(P_{h} \rho_{t}, \theta(t)\right)$, or, noting that $A\left(\theta_{t}, \theta\right)=\left(\frac{d}{d t}\right) A(\theta, \theta) / 2$, after integration over $(0, T)$,

$$
\begin{aligned}
&\|\theta(T)\|_{1}^{2}+\int_{0}^{T} \int_{0}^{t} \beta(t-s)\left(A_{h} \theta(s), A_{h} \theta(t)\right) d s d t \\
& \leq C\left\{\|\theta(0)\|_{1}^{2}+\int_{0}^{T}\left\|P_{h} \rho_{t}\right\|_{1}\|\theta\|_{1} d t\right\} .
\end{aligned}
$$

By Lemma 2.1, applied with $H=S_{h}$ and $B=A_{h}^{2}$, the double integral is nonnegative, and we conclude easily

$$
\|\theta(T)\|_{1} \leq C\left\{\|\theta(0)\|_{1}+\int_{0}^{T}\left\|P_{h} \rho_{t}\right\|_{1} d t\right\}, \quad \text { for } T \geq 0,
$$

whence, using also the assumed boundedness of $P_{h}$ in $H^{1}$,

$$
\|\theta(T)\|_{1} \leq C\left\{\left\|v_{h}-v\right\|_{1}+\|\rho(0)\|_{1}+\int_{0}^{T}\left\|\rho_{t}\right\|_{1} d t\right\}, \quad \text { for } T \geq 0 .
$$

An application of (2.4) now completes the proof.

We remark that a sufficient condition for the $L_{2}$-projection $P_{h}$ to be bounded in $H^{1}(\Omega)$ is the standard inverse inequality

$$
\|\chi\|_{1} \leq C h^{-1}\|\chi\|, \quad \forall \chi \in S_{h}
$$

weaker conditions have been given in Crouzeix and Thomée [4].

We shall complete this section by citing some previous work on the spatially semidiscrete solution of equations of type (2.1).

Neta [15] treats a nonlinear equation, with $\beta$ smooth and $A u$ replaced by the one-dimensional operator $\sigma\left(u_{x}\right)_{x}(\Omega=(0,1))$ where $\sigma^{\prime}$ is positive, and claims an error estimate of the form

$$
\left\|u_{h}-u\right\|_{L_{2}\left(H^{\prime}\right)} \leq C(u) h^{r-1} .
$$


However, his argument uses an assumption which is incorrectly stated to be a generalisation of a known property for positive definite kernels in the case when $\sigma^{\prime}$ is constant, and which is not satisfied for simple kernels.

Jin Choi and MacCamy [2] consider the weakly-singular kernel $\beta(t)=$ $c t^{\alpha-1} e^{-t}$, with $0<\alpha<1$, where the exponential is included in order that $\beta \in L_{1}\left(\mathbf{R}_{+}\right)$. Letting $\tilde{H}^{\alpha}$ denote the Hilbert space defined by

$$
\|u\|_{\tilde{H}^{\alpha}}=\left(\|u\|_{H^{1 / 2}\left(L_{2}\right)}^{2}+\|u\|_{H^{-\alpha / 2}\left(H^{\prime}\right)}^{2}\right)^{1 / 2}, \quad \text { with } H^{s}(V)=H^{s}\left(\mathbf{R}_{+} ; V(\Omega)\right),
$$

where the fractional-order Sobolev norms in time are defined in terms of Laplace transforms, they show that

$$
\left\|u_{h}-u\right\|_{\tilde{H}^{\alpha}} \leq C \inf _{\chi \in H^{1 / 2}\left(S_{h}\right)}\|\chi-u\|_{\tilde{H}^{\alpha}}
$$

i.e., that the semidiscrete solution is optimal with respect to $\tilde{H}^{\alpha}$, thus extending a result from Douglas and Dupont [6] for the parabolic case $(\alpha=0)$. As a result,

$$
\left\|u_{h}-u\right\|_{\bar{H}^{\alpha}} \leq C h\left(\|u\|_{H^{1 / 2}\left(H^{\prime}\right)}+\|u\|_{H^{-\alpha / 2}\left(H^{2}\right)}\right) \text {. }
$$

They also demonstrate that for this kernel

$$
\left\|u_{h}-u\right\|_{L_{2}\left(L_{2}\right)} \leq C h^{2}\|u\|_{L_{2}\left(H^{2}\right)}
$$

which result is based on, in our above notation, the inequality

$$
\|\theta\|_{L_{2}\left(L_{2}\right)} \leq C\|\rho\|_{L_{2}\left(L_{2}\right)}
$$

\section{Discretisation in time}

We shall now consider the discretisation in time of the spatially semidiscrete problem (2.1) studied above. Introducing the time step $k$, setting $t_{n}=n k$, and letting $U^{n} \in S_{h}$ be the approximation of $u_{h}\left(t_{n}\right)$, we shall first consider discretisations in which the time derivative is replaced by the backward difference quotient $\bar{\partial}_{t} U^{n}=\left(U^{n}-U^{n-1}\right) / k$. Using a quadrature formula

$$
q_{n}(\varphi)=\sum_{j=0}^{n} \omega_{n j} \varphi^{j} \approx \int_{0}^{t_{n}} \beta\left(t_{n}-s\right) \varphi(s) d s, \quad \text { with } \varphi^{j}=\varphi\left(t_{j}\right)
$$


to handle the integral term, we then arrive at the fully discrete problem

$$
\begin{aligned}
\left(\bar{\partial}_{t} U^{n}, \chi\right)+q_{n}(A(U, \chi)) & =\left(f_{n}, \chi\right), \quad \text { for } n \geq 1, \text { with } f_{n}=f\left(t_{n}\right), \\
U^{0} & =v_{h} .
\end{aligned}
$$

Later, we shall also analyse schemes with second-order approximation of the time derivative.

We introduce the quadrature error

$$
\epsilon_{n}(\varphi)=q_{n}(\varphi)-\int_{0}^{t_{n}} \beta\left(t_{n}-s\right) \varphi(s) d s,
$$

and say that $q_{n}$ is accurate of order $p$ if $\epsilon_{n}(\varphi)=O\left(k^{p}\right)$ for $\varphi$ sufficiently regular. In our estimates below, we shall use the "global quadrature error"

$$
\tilde{\epsilon}_{N}(\varphi)=k \sum_{n=1}^{N}\left\|\epsilon_{n}(\varphi)\right\|, \quad \text { for } t_{N} \leq T ;
$$

when $\varphi \in C\left([0, T], L_{2}(\Omega)\right)$. Precise estimates for this quantity are given in Section 4, where we discuss specific choices for $q_{n}$.

In order to show an error estimate for (3.2) we shall need a stability result. For this purpose, as we mentioned in the discussion of stability in Section 1, it is natural to assume that for the quadratic form analogous to the double integral in (1.2) we have

$$
Q_{N}(\Phi)=k \sum_{n=1}^{N} q_{n}(\Phi) \Phi^{n} \geq 0, \quad \forall \Phi=\left(\Phi^{0}, \ldots, \Phi^{N}\right)^{\top}
$$

We shall term such a quadrature rule $q_{n}$ positive. We note that, in general, if some of the $\omega_{n 0}$ are nonzero, (3.5) cannot hold since $Q_{N}(\Phi)$ lacks a quadratic term in $\Phi_{0}$. However, in our first result we may assume that $\omega_{n 0}=0$ for $n \geq 1$, since the quadrature rules of first order that we shall propose in Section 4 have this property.

We note that $\omega_{n n}>0$ for $n \geq 1$ is a necessary condition for $q_{n}$ to be positive. This means that the matrix coefficient of $U^{n}$ in (3.2) contains a term in the stiffness matrix corresponding to $A$; in particular, this equation is implicit.

We are now ready to present our stability result for (3.2).

LEMMA 3.1. Assume that $\beta$ is positive definite and that $q_{n}$ is positive. Then the solution of (3.2) satisfies

$$
\left\|U^{N}\right\| \leq\left\|v_{h}\right\|+2 k \sum_{n=1}^{N}\left\|f_{n}\right\|, \quad \text { for } N \geq 1 .
$$


PROOF. Setting $\chi=U^{n}$ in (3.2) and noting that $q_{n}(A(U, \chi))=A\left(q_{n}(U), \chi\right)$ we have

$$
\frac{1}{2} \bar{\partial}_{t}\left\|U^{n}\right\|^{2}+\frac{k}{2}\left\|\bar{\partial}_{t} U^{n}\right\|^{2}+A\left(q_{n}(U), U^{n}\right)=\left(f_{n}, U^{n}\right), \quad \text { for } n \geq 1,
$$

so that, with $A_{h}$ defined by (2.9),

$$
\left\|U^{n}\right\|^{2}-\left\|U^{n-1}\right\|^{2}+2 k\left(q_{n}\left(A_{h}^{1 / 2} U\right), A_{h}^{1 / 2} U^{n}\right) \leq 2 k\left\|U^{n}\right\|\left\|f_{n}\right\| .
$$

Since the quadrature formula is positive,

$$
\sum_{n=1}^{N} 2 k\left(q_{n}\left(A_{h}^{1 / 2} U\right), A_{h}^{\mathrm{i} / 2} U^{n}\right)=2 \int_{\Omega} Q_{N}\left(A_{h}^{1 / 2} U\right) d x \geq 0,
$$

and so, after summation of (3.6),

$$
\left\|U^{N}\right\|^{2}-\left\|v_{h}\right\|^{2} \leq 2 k \sum_{n=1}^{N}\left\|U^{n}\right\|\left\|f_{n}\right\|, \quad \forall N \geq 1 .
$$

Now, with $M$ chosen so that $\left\|U^{M}\right\|=\max _{0 \leq j \leq N}\left\|U^{j}\right\|$, we have

$$
\begin{aligned}
\left\|U^{N}\right\|^{2} \leq\left\|U^{M}\right\|^{2} & \leq\left\|v_{h}\right\|^{2}+2 k \sum_{n=1}^{M}\left\|U^{n}\right\|\left\|f_{n}\right\| \\
& \leq\left\{\left\|v_{h}\right\|+2 k \sum_{n=1}^{M}\left\|f_{n}\right\|\right\}\left\|U^{M}\right\|,
\end{aligned}
$$

from which the result follows.

We can now state and prove our error estimate for (3.2). It shows an $O\left(h^{r}+k\right)$ convergence rate provided the solution of (1.1) is sufficiently smooth and $q_{n}$ is first order accurate.

THEOREM 3.1. Assume that $\beta$ is positive definite, and that $\left\{S_{h}\right\}$ satisfies (1.5). If $q_{n}$ is positive and if $v_{h}$ is chosen so that

$$
\left\|v_{h}-v\right\| \leq C h^{r}\|v\|_{r},
$$


then for the solutions of (3.2) and (1.1) we have

$$
\begin{aligned}
\left\|U^{N}-u\left(t_{N}\right)\right\| \leq & C h^{r}\left\{\|v\|_{r}+\int_{0}^{t_{N}}\left\|u_{t}\right\|_{r} d s\right\} \\
& +C k \int_{0}^{t_{N}}\left\|u_{t}\right\| d s+2 \tilde{\epsilon}_{N}(A u), \quad \text { for } t_{N} \geq 0 .
\end{aligned}
$$

PROOF. Using the elliptic projection $R_{h}: H_{0}^{1}(\Omega) \rightarrow S_{h}$ defined in (2.3) we write

$$
e^{n}=U^{n}-u\left(t_{n}\right)=\left(U^{n},-R_{h} u\left(t_{n}\right)\right)+\left(R_{h} u\left(t_{n}\right)-u\left(t_{n}\right)\right) \equiv \theta^{n}+\rho^{n} .
$$

By (2.4) we have

$$
\left\|\rho^{n}\right\| \leq C h^{r}\left\|u\left(t_{n}\right)\right\|_{r} \leq C h^{r}\left\{\|v\|_{r}+\int_{0}^{t_{n}}\left\|u_{r}\right\|_{r} d s\right\} .
$$

For $\theta^{n}$ we obtain by our definitions that

$$
\begin{aligned}
\left(\bar{\partial}_{t} \theta^{n}, \chi\right)+A & \left(q_{n}(\theta), \chi\right) \\
& =\left(\bar{\partial}_{t} U^{n}, \chi\right)+A\left(q_{n}(U), \chi\right)-\left(\bar{\partial}_{t} R_{h} u_{n}, \chi\right)-A\left(q_{n}\left(R_{h} u\right), \chi\right) \\
& =\left(f_{n}, \chi\right)-\left(\bar{\partial}_{t} u_{n}, \chi\right)-\left(\bar{\partial}_{t} \rho^{n}, \chi\right)-A\left(q_{n}(u), \chi\right) \\
& =\left(u_{t}\left(t_{n}\right)-\bar{\partial}_{t} u_{n}, \chi\right)+\int_{0}^{t_{n}} \beta\left(t_{n}-s\right) A(u(s), \chi) d s \\
& -\left(q_{n}(A u), \chi\right)-\left(\bar{\partial}_{t} \rho^{n}, \chi\right) .
\end{aligned}
$$

Thus, letting

$$
\begin{aligned}
\tau^{n} & \equiv \tau_{1}^{n}+\tau_{2}^{n}+\tau_{3}^{n} \\
& \equiv\left\{u_{t}\left(t_{n}\right)-\bar{\partial}_{t} u\left(t_{n}\right)\right\}+\left\{\int_{0}^{t_{n}} \beta\left(t_{n}-s\right) A u(s) d s-q_{n}(A u)\right\}-\bar{\partial}_{\imath} \rho^{n},
\end{aligned}
$$

we have

$$
\left(\bar{\partial}_{t} \theta^{n}, \chi\right)+A\left(q_{n}(\theta), \chi\right)=\left(\tau^{n}, \chi\right),
$$

and so, applying Lemma 3.1,

$$
\left\|\theta^{N}\right\| \leq\left\|\theta^{0}\right\|+2 k \sum_{n=1}^{N}\left\|\tau^{n}\right\| .
$$

Here,

$$
\left\|\theta^{0}\right\|=\left\|v_{h}-R_{h} v\right\| \leq\left\|v_{h}-v\right\|+\left\|R_{h} v-v\right\| \leq C h^{r}\|v\|_{r}
$$


Further

$$
k \sum_{n=1}^{N}\left\|\tau_{1}^{n}\right\| \leq C k \sum_{n=1}^{N} \int_{t_{n-1}}^{t_{n}}\left\|u_{t t}\right\| d s=C k \int_{0}^{t_{N}}\left\|u_{t t}\right\| d s
$$

and

$$
k \sum_{n=1}^{N}\left\|\tau_{3}^{n}\right\| \leq \sum_{n=1}^{N} \int_{t_{n-1}}^{t_{n}}\left\|\rho_{t}\right\| d s \leq C h^{r} \int_{0}^{t_{N}}\left\|u_{t}\right\|_{r} d s .
$$

Together these estimates complete the proof, because $\tau_{2}^{n}=-\epsilon_{n}(A u)$.

Since the backward difference quotient used above to approximate $u_{t}$ is only first-order accurate, we now try to obtain higher accuracy by employing instead a second order backward difference operator. Thus set

$$
D_{t}^{(2)} U^{n}=\bar{\partial}_{t} U^{n}+\frac{1}{2} k \bar{\partial}_{t}^{2} U^{n}
$$

and let $U^{n}, n=0,1, \ldots$, be defined by

$$
\begin{aligned}
\left(D_{t}^{(2)} U^{n}, \chi\right)+q_{n}(A(U, \chi)) & =\left(f_{n}, \chi\right), \quad \text { for } n \geq 2, \\
\left(\bar{\partial}_{t} U^{1}, \chi\right)+q_{1}(A(U, \chi)) & =\left(f_{1}, \chi\right), \\
U^{0} & =v_{h} .
\end{aligned}
$$

Notice that since the second-order backward difference quotient can only be applied for $n \geq 2$, we have had to use a separate equation for $n=1$.

Since we now need a second-order quadrature rule, we shall need to include in it a term in $\Phi^{0}$. For this reason, as mentioned earlier, (3.5) will not be satisfied, and we shall have to modify our requirements on $q_{n}$. Thus we say that $q_{n}$ is weakly positive if $Q_{N}(\Phi) \geq 0$ for all $\Phi$ with $\Phi^{0}=0$. This will suffice in the analysis when $v_{h}$ is chosen as $R_{h} v$. In order to treat more general choices, we say that $q_{n}$ is $\omega_{0}$-positive if

$$
Q_{N}(\Phi) \geq-\omega_{0}\left(\Phi^{0}\right)^{2}, \quad \forall N \geq 1, \Phi=\left(\Phi^{0}, \ldots, \Phi^{N}\right)^{\top} .
$$

Note that 0 -positive is the same as positive, and that $\omega_{n n}>0$ for $n \geq 1$ is necessary for weak positivity.

We now have the following stability result.

LEMMA 3.2. Assume that $\beta$ is positive definite and that $q_{n}$ is $\omega_{0}$-positive. Then the solution of (3.7) satisfies

$$
\left\|U^{N}\right\| \leq\left\|v_{h}\right\|+3 \omega_{0}^{1 / 2}\left\|A_{h}^{1 / 2} v_{h}\right\|+3 k \sum_{n=1}^{N}\left\|f_{n}\right\|, \quad \text { for } N \geq 1 .
$$


If $q_{n}$ is weakly positive, then

$$
\left\|U^{N}\right\| \leq 3 k \sum_{n=1}^{N}\left\|f_{n}\right\|, \quad \text { for } N \geq 1, \quad \text { when } v_{h}=0 .
$$

PROOF. We prove the first statement. The proof of the second follows at once by setting $U^{0}=0$ in the argument. With $\Delta_{k} U^{n}=U^{n}-U^{n-k}$ for $k=1,2$, we may write

$$
k D_{t}^{(2)} U^{n}=\frac{3}{2} U^{n}-2 U^{n-1}+\frac{1}{2} U^{n-2}=2 \Delta_{1} U^{n}-\frac{1}{2} \Delta_{2} U^{n} .
$$

Since

$$
2\left(\Delta_{k} U^{n}, U^{n}\right)=\Delta_{k}\left\|U^{n}\right\|^{2}+\left\|\Delta_{k} U^{n}\right\|^{2},
$$

we have

$k\left(D_{t}^{(2)} U^{n}, U^{n}\right)=\Delta_{1}\left\|U^{n}\right\|^{2}-\frac{1}{4} \Delta_{2}\left\|U^{n}\right\|^{2}+\left\|\Delta_{1} U^{n}\right\|^{2}-\frac{1}{4}\left\|\Delta_{2} U^{n}\right\|^{2}, \quad$ for $n \geq 2$.

By summation from 2 to $N$,

$$
\sum_{n=2}^{N}\left(\Delta_{1}\left\|U^{n}\right\|^{2}-\frac{1}{4} \Delta_{2}\left\|U^{n}\right\|^{2}\right)=\frac{3}{4}\left\|U^{N}\right\|^{2}-\frac{1}{4}\left\|U^{N-1}\right\|^{2}-\frac{3}{4}\left\|U^{1}\right\|^{2}+\frac{1}{4}\left\|U^{0}\right\|^{2},
$$

and further, since $\Delta_{2} U^{n}=\Delta_{1} U^{n}+\Delta_{1} U^{n-1}$, we obtain

$$
\begin{aligned}
\sum_{n=2}^{N}\left(\left\|\Delta_{1} U^{n}\right\|^{2}-\frac{1}{4}\left\|\Delta_{2} U^{n}\right\|^{2}\right) & \geq \sum_{n=2}^{N}\left(\left\|\Delta_{1} U^{n}\right\|^{2}-\frac{1}{4}\left(\left\|\Delta_{1} U^{n}\right\|+\left\|\Delta_{1} U^{n-1}\right\|\right)^{2}\right) \\
& \geq \frac{1}{2} \sum_{n=2}^{N}\left(\left\|\Delta_{1} U^{n}\right\|^{2}-\left\|\Delta_{1} U^{n-1}\right\|^{2}\right) \\
& =\frac{1}{2}\left(\left\|\Delta_{1} U^{N}\right\|^{2}-\left\|\Delta_{1} U^{1}\right\|^{2}\right) .
\end{aligned}
$$

Hence,

$$
\begin{aligned}
k\left(\bar{\partial}_{t} U^{1}, U^{1}\right)+ & k \sum_{n=2}^{N}\left(D_{t}^{(2)} U^{n}, U^{n}\right) \\
\geq & \frac{1}{2}\left\{\left\|U^{1}\right\|^{2}-\left\|U^{0}\right\|^{2}+\left\|\Delta_{1} U^{1}\right\|^{2}\right\}+\frac{1}{2}\left\{\left\|\Delta_{1} U^{N}\right\|^{2}-\left\|\Delta_{1} U^{1}\right\|^{2}\right\} \\
& \quad+\left\{\frac{3}{4}\left\|U^{N}\right\|^{2}-\frac{1}{4}\left\|U^{N-1}\right\|^{2}-\frac{3}{4}\left\|U^{1}\right\|^{2}+\frac{1}{4}\left\|U^{0}\right\|^{2}\right\} \\
\geq & \frac{3}{4}\left\|U^{N}\right\|^{2}-\frac{1}{4}\left\|U^{N-1}\right\|^{2}-\frac{1}{4}\left\|U^{1}\right\|^{2}-\frac{1}{4}\left\|U^{0}\right\|^{2}
\end{aligned}
$$


But by (3.7) we have

$$
k\left(\bar{\partial}_{t} U^{1}, U^{1}\right)+k \sum_{n=2}^{N}\left(D_{t}^{(2)} U^{n}, U^{n}\right)+\int_{\Omega} Q_{N}\left(A_{h}^{\frac{1}{2}} U\right) d x=k \sum_{n=1}^{N}\left(f_{n}, U^{n}\right),
$$

and by (3.9) and (3.8) this yields

$$
\left\|U^{N}\right\|^{2} \leq \frac{1}{3}\left\{\left\|U^{N-1}\right\|^{2}+\left\|U^{1}\right\|^{2}+\left\|U^{0}\right\|^{2}\right\}+\frac{4}{3} k \sum_{n=1}^{N}\left(f_{n}, U^{n}\right)+\frac{4}{3} \omega_{0}\left\|A_{h}^{1 / 2} U^{0}\right\|^{2} .
$$

As in the first-order case, suppose $\left\|U^{M}\right\|=\max _{0 \leq n \leq N}\left\|U^{n}\right\|$. Then

$$
\begin{aligned}
\left\|U^{M}\right\|^{2} & \leq \frac{1}{3}\left\{\left\|U^{M-1}\right\|^{2}+\left\|U^{1}\right\|^{2}+\left\|U^{0}\right\|^{2}\right\}+\frac{4}{3} k \sum_{n=1}^{M}\left\|f_{n}\right\|\left\|U^{n}\right\|+\frac{4}{3} \omega_{0}\left\|A_{h}^{1 / 2} U^{0}\right\|^{2} \\
& \leq \frac{1}{3}\left\|U^{M}\right\|^{2}+\frac{1}{3}\left\{\left\|U^{1}\right\|+\left\|U^{0}\right\|+4 k \sum_{n=1}^{N}\left\|f_{n}\right\|\right\}\left\|U^{M}\right\|+\frac{4}{3} \omega_{0}\left\|A_{h}^{1 / 2} U^{0}\right\|^{2}
\end{aligned}
$$

so

$$
\left\|U^{M}\right\|^{2} \leq\left\{\frac{1}{2}\left(\left\|U^{1}\right\|+\left\|U^{0}\right\|\right)+2 k \sum_{n=1}^{N}\left\|f_{n}\right\|\right\}\left\|U^{M}\right\|+2 \omega_{0}\left\|A_{h}^{1 / 2} U^{0}\right\|^{2},
$$

and hence

$$
\begin{aligned}
\left\|U^{M}\right\|^{2} & \leq\left\{\frac{1}{2}\left(\left\|U^{1}\right\|+\left\|U^{0}\right\|\right)+2 k \sum_{n=1}^{N}\left\|f_{n}\right\|\right\}^{2}+4 \omega_{0}\left\|A_{h}^{1 / 2} U^{0}\right\|^{2} \\
& \leq\left\{\frac{1}{2}\left(\left\|U^{1}\right\|+\left\|U^{0}\right\|\right)+2 k \sum_{n=1}^{N}\left\|f_{n}\right\|+2 \omega_{0}^{1 / 2}\left\|A_{h}^{1 / 2} U^{0}\right\|\right\}^{2},
\end{aligned}
$$

from which we conclude that

$$
\left\|U^{N}\right\| \leq \frac{1}{2}\left(\left\|U^{1}\right\|+\left\|v_{h}\right\|\right)+2 k \sum_{n=1}^{N}\left\|f_{n}\right\|+2 \omega_{0}^{1 / 2}\left\|A_{h}^{1 / 2} v_{h}\right\| .
$$

Since

$$
\left(U^{1}-U^{0}, U^{1}\right)+k A\left(q_{1}(U), U^{1}\right)=k\left(f_{1}, U^{1}\right),
$$

we easily obtain, using (3.8) for $N=1$, that

$$
\left\|U^{1}\right\|^{2} \leq\left\{\left\|U^{0}\right\|+k\left\|f_{1}\right\|\right\}\left\|U^{1}\right\|+\omega_{0}\left\|A_{h}^{1 / 2} U^{0}\right\|^{2},
$$


and hence

$$
\left\|U^{1}\right\| \leq\left\|v_{h}\right\|+\left(2 \omega_{0}\right)^{1 / 2}\left\|A_{h}^{1 / 2} v_{h}\right\|+k\left\|f_{1}\right\| .
$$

The proof is now complete.

As a consequence of Lemma 3.2 we have the following error estimate, where again the global quadrature error is defined by (3.4).

THEOREM 3.2. Assume that $\beta$ is positive definite, and that $\left\{S_{h}\right\}$ satisfies (1.5). If $q_{n}$ is weakly positive, then for the solution of (3.7) with $v_{h}=R_{h} v$ we have, for $t_{N}>0$,

$$
\begin{aligned}
&\left\|U^{N}-u\left(t_{N}\right)\right\| \leq C h^{r}\left\{\|v\|_{r}+\int_{0}^{t_{N}}\left\|u_{t}\right\|_{r} d s\right\} \\
&+C k \int_{0}^{2 k}\left\|u_{t t}\right\| d s+C k^{2} \int_{k}^{t_{N}}\left\|u_{t t t}\right\| d s+\tilde{\epsilon}_{N}(A u), \\
& \text { for } t_{N}>0 .
\end{aligned}
$$

If $q_{n}$ is $\omega_{0}$-positive and $v_{h}$ is chosen so that

$$
\left\|v_{h}-v\right\|+h\left\|v_{h}-v\right\|_{1} \leq C h^{r}\|v\|_{r},
$$

then the error estimate (3.10) remains valid after the addition of a term $C h^{r-1} \omega_{0}^{1 / 2}\|v\|_{r}$ to the error bound.

PROOF. We note that for smooth solutions this error estimate is of order $O\left(h^{r}+\right.$ $k^{2}$ ) plus the quadrature error. The form of the terms in $k$ is chosen to accomodate also weakly singular kernels.

The proof parallels that of Theorem 3.1. This time the equation for $\theta^{n}$ for $n \geq 2$ is

$$
\left(D_{t}^{(2)} \theta^{n}, \chi\right)+A\left(q_{n}(\theta), \chi\right)=\left(\tau^{n}, \chi\right),
$$

where $\tau^{n}=\tau_{1}^{n}+\tau_{2}^{n}+\tau_{3}^{n}$ is given by

$$
\tau_{1}^{n}=u_{t}\left(t_{n}\right)-D_{i}^{(2)} u\left(t_{n}\right), \quad \tau_{2}^{n}=-\epsilon_{n}(A u), \quad \tau_{3}^{n}=-D_{t}^{(2)} \rho^{n} .
$$

Treating the first term in the approximation of $u_{t}$ separately we now get

$$
k \sum_{n=2}^{N}\left\|\tau_{1}^{n}\right\| \leq C k \int_{0}^{2 k}\left\|u_{t t}\right\| d s+C k^{2} \int_{k}^{t_{N}}\left\|u_{t u}\right\| d s
$$


and

$$
k \sum_{n=2}^{N}\left\|\tau_{3}^{n}\right\| \leq C h^{r} \int_{0}^{t_{N}}\left\|u_{t}\right\|_{r} d s .
$$

Also, using the estimates for the standard backward Euler method from the proof of Theorem 3.1 for $n=1$ we have

$$
k\left\|\tau_{1}^{1}\right\| \leq k \int_{0}^{k}\left\|u_{t t}\right\| d s \quad \text { and } \quad k\left\|\tau_{3}^{1}\right\| \leq C h^{r} \int_{0}^{k}\left\|u_{t}\right\|_{r} d s .
$$

For the case that $q_{n}$ is positive these estimates complete the proof by Lemma 3.2. In the $\omega_{0}$-positive case we also need to note that after writing $\theta_{0}=\left(v_{h}-v\right)-$ $\left(R_{h} v-v\right)$ we have

$$
\left\|\theta_{0}\right\|+3 \omega_{0}^{1 / 2}\left\|A_{h}^{1 / 2} \theta_{0}\right\| \leq\left\|\theta_{0}\right\|+C \omega_{0}^{1 / 2}\left\|\theta_{0}\right\|_{1} \leq C\left(h^{r}+\omega_{0}^{1 / 2} h^{r-1}\right)\|v\|_{r} .
$$

A possible alternative to (3.7) would be to think of $\bar{\partial}_{t} U^{n}$ in (3.2) as an approximation to $u_{t}\left(t_{n-1 / 2}\right)$, and to approximate the integral with upper limit $t_{n-1 / 2}$ to second-order. As we shall see in Section 4 below, this appears difficult to combine with positivity of $q_{n}$ in the above sense, and suggests a modification in the definition of the quadratic form $Q_{N}(\Phi)$ in this case.

\section{Positive-definite quadrature formulas}

We now turn to a discussion of quadrature formulas suitable for application in the difference schemes proposed above. We recall the notation

$$
q_{n}(\Phi)=\sum_{j=0}^{n} \omega_{n j} \Phi^{j}, \quad \text { for } n \geq 1,
$$

and

$$
Q_{N}(\Phi)=k \sum_{n=1}^{N} q_{n}(\Phi) \Phi^{n}=k \sum_{n=1}^{N} \sum_{j=0}^{n} \omega_{n j} \Phi^{j} \Phi^{n} .
$$

We are interested in conditions for $\boldsymbol{q}_{n}$ to satisfy our various positivity properties introduced in Section 3, and also in giving bounds for the global quadrature error $\tilde{\epsilon}_{N}(A u)$ appearing in the error estimates of Theorems 3.1 and 3.2. We first show the following lemma which is relevant for the case that the quadrature formula is of convolution type. 
LEMMA 4.1. Let $\left\{b_{j}\right\}_{j=0}^{\infty} \in l_{1}$ be a sequence of positive numbers such that

$$
\tilde{b}(\theta)=\sum_{j=0}^{\infty} b_{j} \cos j \theta \geq 0, \quad \text { for } \theta \in \mathbf{R} .
$$

Then

$$
B_{N}(\Phi)=\sum_{n=1}^{N} \sum_{j=1}^{n} b_{n-j} \Phi^{j} \Phi^{n} \geq 0, \quad \forall \Phi=\left(\Phi^{1}, \ldots, \Phi^{N}\right) \in \mathbf{R}^{N}, \quad N \geq 1 .
$$

PROOF. Letting ^ denote the Fourier transform, so that, e.g.

$$
\hat{b}(\theta)=\sum_{j=0}^{\infty} b_{j} e^{i j \theta}
$$

we have, by a simple calculation, with $\Phi^{j}=0$ for $j \notin[1, N]$,

$$
B_{N}(\Phi)=\frac{1}{2 \pi} \int_{0}^{2 \pi} \hat{b}(\theta)|\hat{\Phi}(\theta)|^{2} d \theta=\frac{1}{2 \pi} \int_{0}^{2 \pi} \operatorname{Re} \hat{b}(\theta)|\hat{\Phi}(\theta)|^{2} d \theta
$$

where the latter equality follows since $B_{N}(\Phi)$ is real-valued. Since $\operatorname{Re} \hat{b}=\tilde{b} \geq$ 0 , this shows the result.

It follows, in particular, from Lemma 4.1 that if (4.2) holds and $\omega_{n j}=b_{n-j}$, then the quadrature formula $q_{n}$ in (4.1) is weakly positive. Further, if $\omega_{n j}=b_{n-j}$, for $j=1, \ldots, n$, but $\omega_{n 0}=0$, then the quadrature rule is positive. Sufficient conditions for (4.2) will follow from our next lemma:

LEMMA 4.2. Assume that the sequence $\left\{a_{j}\right\}_{j=0}^{\infty} \in l_{1}$ is positive and convex. Then

$$
\frac{1}{2} a_{0}+\sum_{j=1}^{\infty} a_{j} \cos j \theta \geq 0, \quad \forall \theta \in \mathbf{R} .
$$

Proof. See Zygmund [22].

Together, Lemmas 4.1 and 4.2 show the following, where we have replaced the $l_{1}$ assumption by boundedness. 
LEMMA 4.3. Assume that the sequence $\left\{a_{j}\right\}_{j=0}^{\infty} \in l_{\infty}$ is positive and convex, and let $b_{0}=a_{0} / 2, b_{j}=a_{j}$, for $j \geq 1$. Then

$$
\sum_{n=1}^{N} \sum_{j=1}^{n} b_{n-j} \Phi^{j} \Phi^{n} \geq 0, \quad \forall\left(\Phi^{1}, \ldots, \Phi^{N}\right) \in \mathbf{R}^{N}, \quad N \geq 1 .
$$

PROOF. For any $\rho \in(0,1)$ we find easily that $\left\{a_{j} \rho^{j}\right\}_{j=0}^{\infty} \in l_{1}$ and is convex. Therefore (4.3) holds with $b_{j}$ replaced by $b_{j} \rho^{j}$. The result now follows by letting $\rho \rightarrow 1$.

We shall now consider specific examples of quadrature formulas and discuss their positivity. We shall assume first that the kernel $\beta$ is smooth for $t \geq 0$ and satisfies

$$
\beta \in C^{2}\left(\mathbf{R}_{+}\right), \quad \text { and } \quad(-1)^{j} \beta^{(j)}(t) \geq 0 \quad \text { for } t>0, \quad j=0,1,2 .
$$

As was mentioned in Section 1, this is sufficient for $\beta$ to be a positive definite kernel if, in addition, $\beta \in L_{1}\left(\mathbf{R}_{+}\right)$. Similarly to the proof of Lemma 4.3 , this latter condition may be reduced to requiring $\beta$ integrable at $t=0$. In fact, $\beta_{\epsilon}(t)=\beta(t) e^{-\epsilon t}$ satisfies (4.4) for any $\epsilon>0$, and $\beta_{\epsilon} \in L_{1}\left(\mathbf{R}_{+}\right)$, so that (1.2) holds with $\beta$ replaced by $\beta_{\epsilon}$, and hence also for $\beta$, by letting $\epsilon \rightarrow 0$.

As our first example we consider the rectangle rule using the values of the integrand at the right-hand end-points of the intervals $\left(t_{j-1}, t_{j}\right)$. This corresponds to taking

$$
q_{n}(\Phi)=k \sum_{j=1}^{n} \beta_{n-j} \Phi^{j}, \quad \text { with } \beta_{j}=\beta\left(t_{j}\right),
$$

so that $\omega_{n 0}=0$ and $\omega_{n j}=k \beta_{n-j}$ for $j=1, \ldots, n$.

LEMMA 4.4. Assume that $\beta \in C([0, T])$ and that (4.4) holds. Then the integration rule (4.5) is positive. Further, if $\beta \in C^{1}([0, T])$, we have for the corresponding global quadrature error defined in (3.4)

$$
\tilde{\epsilon}_{N}(\varphi) \leq C_{T} k\left\{\|\varphi(0)\|+\int_{0}^{t_{N}}\left\|\varphi_{i}\right\| d s\right\}, \quad \text { for } t_{N} \leq T .
$$

ProOF. As a result of (4.4) the sequence $\left\{\beta_{j}\right\}_{j=0}^{\infty}$ is positive, bounded, and convex. The assumptions of Lemma 4.3 are therefore satisfied for $a_{j}=\beta_{j}$, and so, with 
$b_{0}=\beta_{0} / 2, b_{j}=\beta_{j}$ for $j \geq 1$, we have

$$
Q_{N}(\Phi)=k^{2}\left\{\sum_{n=1}^{N} b_{0}\left(\Phi^{n}\right)^{2}+\sum_{n=1}^{N} \sum_{j=1}^{n} b_{n-j} \Phi^{j} \Phi^{n}\right\} \geq \frac{1}{2} k^{2} \beta_{0} \sum_{n=1}^{N}\left(\Phi^{n}\right)^{2} \geq 0,
$$

for all $\Phi$.

The quadrature error (3.3) satisfies

$$
\left|\epsilon_{n}(\varphi)\right| \leq C k \int_{0}^{t_{n}}\left|\frac{\partial}{\partial s}\left[\beta\left(t_{n}-s\right) \varphi(s)\right]\right| d s,
$$

for each $x \in \Omega$, and therefore an application of Minkowski's inequality yields

$$
\left\|\epsilon_{n}(\varphi)\right\| \leq C k \int_{0}^{t_{n}}\left(\|\varphi\|+\left\|\varphi_{t}\right\|\right) d s \leq C k\left\{\|\varphi(0)\|+\int_{0}^{t_{N}}\left\|\varphi_{t}\right\| d s\right\},
$$

which implies the required estimate by summation over $n$.

Combination of Theorem 3.1 and Lemma 4.4 thus shows that for the backward Euler approximation, combined with the present quadrature formula, we have, for $t_{N} \leq T$,

$$
\begin{aligned}
\left\|U^{N}-u\left(t_{N}\right)\right\| \leq C h^{r} & \left\{\|v\|_{r}+\int_{0}^{t_{N}}\left\|u_{t}\right\|_{r} d s\right\} \\
& +C_{T} k\left\{\|v\|_{2}+\int_{0}^{t_{N}}\left(\left\|u_{t t}\right\|+\left\|u_{t}\right\|_{2}\right) d s\right\} .
\end{aligned}
$$

We recall from Section 3 that a weakly-positive quadrature rule has to be implicit in the sense that $\omega_{n n}>0$ for $n \geq 1$. In particular, therefore, the rectangle rule using the values of the functions at the left- rather than the righthand end-points of the intervals $\left(t_{j-1}, t_{j}\right)$ does not satisfy the required weak positivity.

Next, we consider the trapezoidal rule for approximating the integral, i.e.,

$$
q_{n}(\Phi)=k\left\{\frac{1}{2} \beta_{0} \Phi^{n}+\sum_{j=1}^{n-1} \beta_{n-j} \Phi^{j}+\frac{1}{2} \beta_{n} \Phi^{0}\right\}, \quad \text { with } \beta_{j}=\beta\left(t_{j}\right) .
$$

LEMMA 4.5. Assume $\beta \in C([0, T])$ and that (4.4) holds. Then the integration rule (4.7) is $\omega_{0}$-positive with $\omega_{0}=k^{2} \beta_{0} / 4$. Further, provided $\beta \in C^{2}([0, T])$, we have

$$
\tilde{\epsilon}_{N}(\varphi) \leq C_{T} k^{2}\left\{\|\varphi(0)\|+\left\|\varphi_{t}(0)\right\|+\int_{0}^{t_{N}}\left\|\varphi_{t}\right\| d s\right\}, \quad \text { for } t_{N} \leq T .
$$


PROOF. Setting $b_{0}=\beta_{0} / 2$ and $b_{j}=\beta_{j}$, for $j>0$, (4.7) may be written

$$
q_{n}(\Phi)=k\left\{\sum_{j=1}^{n} b_{n-j} \Phi^{j}+\frac{1}{2} b_{n} \Phi^{0}\right\}
$$

We therefore obtain

$$
Q_{N}(\Phi)=k^{2}\left\{\sum_{n=1}^{N} \sum_{j=1}^{n} b_{n-j} \Phi^{j} \Phi^{n}+\frac{1}{2} \sum_{n=1}^{N} b_{n} \Phi^{0} \Phi^{n}\right\},
$$

or, after a simple rearrangement,

$$
Q_{N}(\Phi)=\frac{1}{2} k^{2}\left\{\sum_{n=0}^{N} \sum_{j=0}^{n} b_{n-j} \Phi^{j} \Phi^{n}+\sum_{n=1}^{N} \sum_{j=1}^{n} b_{n-j} \Phi^{j} \Phi^{n}-b_{0}\left(\Phi^{0}\right)^{2}\right\}
$$

Since $\beta$ satisfies (4.4), application of Lemma 4.3 shows that the two sums inside the brackets in (4.8) are nonnegative (the first after a simple shift of the indices). Hence we conclude that $q_{n}$ is $\omega_{0}$-positive with $\omega_{0}=k^{2} b_{0} / 2=k^{2} \beta_{0} / 4$.

The quadrature error is handled by repeating the argument used for the corresponding estimate in Lemma 4.4, except that now, instead of (4.6), we have

$$
\left|\epsilon_{n}(\varphi)\right| \leq C k^{2} \int_{0}^{t_{N}}\left|\left(\frac{\partial}{\partial s}\right)^{2}\left[\beta\left(t_{n}-s\right) \varphi(s)\right]\right| d s .
$$

For the second-order backward-differencing scheme (3.7), using the trapezoidal rule (4.7), Lemma 4.5 and Theorem 3.2 thus yield an error estimate which is $O\left(h^{r}+k^{2}\right)$ if $v_{h}=R_{h} v$, and which for a general $v_{h}$ satisfying (3.11) contains an additional term of order $O\left(k h^{r-1}\right)$.

A perhaps more obvious way than the backward-differencing scheme (3.7) discussed above to attain second-order accuracy for the integro-differential equation would be to consider $\bar{\partial}_{t} U^{n}$ as a second-order approximation to $u_{t}\left(t_{n-\frac{1}{2}}\right)$, as in the Crank-Nicolson scheme for the heat equation, and then replace the integral by an approximation to second order of

$$
\int_{0}^{t_{n-\frac{1}{2}}^{2}} \beta\left(t_{n-\frac{1}{2}}-s\right) A u(s) d s
$$

or

$$
\frac{1}{2}\left\{\int_{0}^{t_{n}} \beta\left(t_{n}-s\right) A u(s) d s+\int_{0}^{t_{n-1}} \beta\left(t_{n-1}-s\right) A u(s) d s\right\} .
$$


However, such a procedure does not necessarily yield the positivity required in our above framework. In order to demonstrate this, we consider the quadrature formula obtained by approximation of the integrals over $\left[0, t_{n-1}\right]$ and $\left[0, t_{n}\right]$ by means of the trapezoidal rule, i.e.,

$$
q_{1}(\Phi)=\frac{1}{2} k\left\{\frac{1}{2} \beta_{0} \Phi^{1}+\frac{1}{2} \beta_{1} \Phi^{0}\right\}
$$

and, for $n \geq 2$,

$$
\begin{aligned}
& q_{n}(\Phi)=\frac{1}{2} k\left\{\frac{1}{2} \beta_{0} \Phi^{n}+\sum_{j=1}^{n-1} \beta_{n-j} \Phi^{j}+\frac{1}{2} \beta_{n} \Phi^{0}\right. \\
& \left.+\frac{1}{2} \beta_{0} \Phi^{n-1}+\sum_{j=1}^{n-2} \beta_{n-1-j} \Phi^{j}+\frac{1}{2} \beta_{n-1} \Phi^{0}\right\} \\
& =\frac{1}{2} k\left\{\frac{1}{2} \beta_{0} \Phi^{n}+\left(\beta_{1}+\frac{1}{2} \beta_{0}\right) \Phi^{n-1}\right. \\
& \left.+\sum_{j=1}^{n-2}\left(\beta_{n-j}+\beta_{n-1-j}\right) \Phi^{j}+\frac{1}{2}\left(\beta_{n-1}+\beta_{n}\right) \Phi^{0}\right\} .
\end{aligned}
$$

This $q_{n}$ is not weakly positive. In fact, weak positivity would require, in particular, $Q_{2}(\Phi)$ to be nonnegative for all $\Phi=\left(0, \Phi^{1}, \Phi^{2}\right)$, or, equivalently, the matrix

$$
\left(\begin{array}{cc}
\beta_{0} & \beta_{1}+\frac{1}{2} \beta_{0} \\
\beta_{1}+\frac{1}{2} \beta_{0} & \beta_{0}
\end{array}\right)=\beta_{0}\left(\begin{array}{cc}
1 & \beta_{1} / \beta_{0}+1 / 2 \\
\beta_{1} / \beta_{0}+1 / 2 & 1
\end{array}\right)
$$

to be positive semidefinite. But, since $\beta_{1}=\beta(k) \rightarrow \beta(0)=\beta_{0}$ as $k \rightarrow 0$, this is impossible for small $\mathrm{k}$ as the limiting matrix is indefinite.

In spite of this, the scheme just discussed will yield a viable method of order $O\left(h^{r}+k^{2}\right)$, as will follow from the following stability estimate. Its proof suggests a modification of the definition of positivity of the quadrature rule for the present case. We note that the scheme under consideration may be written as

$$
\begin{aligned}
\left(\bar{\partial}_{t} U^{n}, \chi\right)+q_{n-1 / 2}(A(U, \chi)) & =\left(f_{n-1 / 2}, \chi\right), \quad \text { for } n \geq 1, \\
U^{0} & =v_{h},
\end{aligned}
$$

with $f_{n-1 / 2}=f\left(t_{n-1 / 2}\right)$ and

$$
\begin{aligned}
q_{1 / 2}(\Phi) & =q_{1}(\Phi) / 2, \\
q_{n-1 / 2}(\Phi) & =\left[q_{n}(\Phi)+q_{n-1}(\Phi)\right] / 2, \quad \text { for } n \geq 2,
\end{aligned}
$$


where $q_{n}$ now denotes the trapezoidal rule (4.7).

LEMMA 4.6. If $\beta \in C[0, T]$ and (4.4) holds, then the solution of the CrankNicolson scheme defined by (4.7), (4.9) and (4.10) satisfies

$$
\left\|U^{N}\right\| \leq\left\|v_{h}\right\|+\frac{1}{4} \beta(0) k^{2}\left\|A_{h} v_{h}\right\|+2 k \sum_{n=1}^{N}\left\|f_{n-1 / 2}\right\|, \quad \text { for } N \geq 1 .
$$

PROOF. Let $\bar{U}^{n}=\left(U^{n}+U^{n-1}\right) / 2$ for $n \geq 1$, so that with $\chi=\bar{U}^{n}$ in (4.9) we obtain

$$
\left\|U^{n}\right\|^{2}-\left\|U^{n-1}\right\|^{2}+2 k\left(q_{n-1 / 2}\left(A_{h} U\right), \bar{U}^{n}\right)=2 k\left(f_{n-1 / 2}, \bar{U}^{n}\right) .
$$

Put $\bar{U}^{0}=0$, and observe that

$$
q_{n-1 / 2}(U)=q_{n}(\tilde{U})-\frac{k}{8}\left(\beta_{n-1}-\beta_{n}\right) U^{0}, \quad \text { for } n \geq 1,
$$

so

$2 k \sum_{n=1}^{N}\left(q_{n-1 / 2}\left(A_{h} U\right), \bar{U}^{n}\right)=2 \int_{\Omega} Q_{N}\left(A_{h}^{1 / 2} \bar{U}\right) d x-\frac{k^{2}}{4} \sum_{n=1}^{N}\left(\beta_{n-1}-\beta_{n}\right)\left(A_{h} U^{0}, \bar{U}\right)$.

By Lemma 4.5 , the trapezoidal rule $q_{n}$ is weakly positive, and hence after summing over $n=1, \ldots, N$ in (4.11), we arrive at

$$
\left\|U^{N}\right\|^{2} \leq\left\|U^{0}\right\|^{2}+\frac{k^{2}}{4}\left\|A_{h} U^{0}\right\| \sum_{n=1}^{N}\left|\beta_{n-1}-\beta_{n}\right|\left\|\bar{U}^{n}\right\|+2 k \sum_{n=1}^{N}\left\|f_{n-1 / 2}\right\|\left\|\bar{U}^{n}\right\| .
$$

Since (4.4) implies

$$
\sum_{n=1}^{N}\left|\beta_{n-1}-\beta_{n}\right|=\sum_{n=1}^{N}\left(\beta_{n-1}-\beta_{n}\right)=\beta_{0}-\beta_{N} \leq \beta_{0},
$$

if we choose $M$ so that $\left\|U^{M}\right\|=\max _{0 \leq n \leq N}\left\|U^{n}\right\|$, then

$$
\left\|U^{M}\right\|^{2} \leq\left\{\left\|U^{0}\right\|+\frac{1}{4} \beta_{0} k^{2}\left\|A_{h} U^{0}\right\|+2 k \sum_{n=1}^{N}\left\|f_{n-1 / 2}\right\|\right\}\left\|U^{M}\right\|,
$$

and the result follows at once. 
Applying this stability estimate to $\theta^{n}=U^{n}-R_{h} u\left(t_{n}\right)$, as in the proof of Theorem 3.1, we see that if $\beta$ is smooth and if $v_{h}=R_{h} v$, then

$$
\left\|\theta^{N}\right\| \leq 2 k \sum_{n=1}^{N}\left\|\tau^{n}\right\|,
$$

where $\rho^{n}=R_{h} u\left(t_{n}\right)-u\left(t_{n}\right)$ and

$\tau^{n}=\left\{u_{t}\left(t_{n}\right)-\bar{\partial}_{t} u\left(t_{n}\right)\right\}+\left\{\int_{0}^{t_{n-1 / 2}} \beta\left(t_{n-1 / 2}-s\right) A u(s) d s-q_{n-1 / 2}(A u)\right\}-\bar{\partial}_{t} \rho^{n}$.

From this, we easily conclude $\left\|U^{n}-u\left(t_{n}\right)\right\|=O\left(h^{r}+k^{2}\right)$, as stated. We shall not pursue this line of investigation in further detail.

One of the difficulties encountered in the numerical solution of integrodifferential equations such as (1.1) by time stepping is that the solution has to be stored at back time levels $t_{j} \leq t_{n}$ in order for the succesive integrals to be approximated. For instance, the difference equation in (1.6) may be written as

$$
\left(1+k \omega_{n n} A_{h}\right) U^{n}+k \sum_{j=1}^{n-1} \omega_{n j} A_{h} U^{j}=U^{n-1}+k P_{h} f_{n}, \quad \text { for } n \geq 1,
$$

where $A_{h}$ is the positive-definite, discrete elliptic operator defined in (2.9), and $P_{h}$ is the $L_{2}$-projection onto $S_{h}$. This difficulty does not occur for standard differential equations, which are local in character and where the solution only has to be retained at a small fixed number of levels. In [17] this problem was addressed and it was proposed, in a similar situation, that the quadrature formula employed be made sparse. We shall now show that such quadrature schemes cannot be expected to be weakly positive.

For this purpose, let us consider a quadrature scheme which uses, as far as possible, the larger time step $k_{1}=m k$, where $m=\left[k^{-1 / 2}\right]$. With $l=l(n)$ the largest integer such that $l k_{1} \leq n k=t_{n}$ we write $\left[0, t_{n}\right]=\left[0, l k_{1}\right] \cup\left[l k_{1}, n k\right]$ and use the trapezoidal rule on $\left[0, l k_{1}\right]$, and the rectangle rule with the right-hand end-point values for $\left[l k_{1}, n k\right]$, i.e.,

$$
\begin{aligned}
\int_{0}^{t_{n}} \varphi(s) d s \approx k_{1}\left[\frac{1}{2} \varphi(0)+\varphi\left(k_{1}\right)+\ldots\right. & \left.+\varphi\left((l-1) k_{1}\right)+\frac{1}{2} \varphi\left(l k_{1}\right)\right] \\
& +k\left[\varphi\left(l k_{1}+k\right)+\ldots+\varphi(n k)\right] .
\end{aligned}
$$

This is a slight modification of a method studied in [17] in that the right-hand rather than the left-hand endpoint values are used-this in order to make the 
method more implicit and thus more stable. This quadrature rule is of order $O\left(k_{1}^{2}+k_{1} k\right)=O(k)$, and thus matches the order of accuracy of the backward Euler discretisation of the time derivative.

In order to see that this quadrature rule is not weakly positive, let $N \equiv$ $1(\bmod m)$. Applying the rule to $\beta\left(t_{n}-s\right) \varphi(s)$ we find that the symmetric matrix corresponding to the quadratic form $Q_{N}(\Phi)$, i.e., $\operatorname{Re}\left(\omega_{N j}\right)=\left(\left(\omega_{N_{j}}\right)+\left(\omega_{j N}\right)\right) / 2$, would contain the $2 \times 2$ principal submatrix, corresponding to $\left(\Phi^{N-1}, \Phi^{N}\right)$,

$$
\left(\begin{array}{cc}
k_{1} \beta_{0} & k_{1} \beta_{1} / 2 \\
k_{1} \beta_{1} / 2 & 2 k \beta_{0}
\end{array}\right)=\frac{1}{2} k_{1} \beta_{0}\left(\begin{array}{cc}
2 & \beta_{1} / \beta_{0} \\
\beta_{1} / \beta_{0} & 4 / m
\end{array}\right) .
$$

Since $\beta_{1} \rightarrow \beta_{0}$ and $m \rightarrow \infty$ as $k \rightarrow 0$, this matrix is indefinite for small $k$, which shows our claim.

We remark that when the kernel has the simple form

$$
\beta(t)=\sum_{l=1}^{M} \gamma_{l} e^{-v_{l} t}, \quad \text { with } \gamma_{l}, \nu_{l}>0, \text { and } M \text { small, }
$$

then the storage problem can be handled easily. For example, in the case of (4.12) corresponding to the right-hand rectangle rule,

$$
\sum_{j=1}^{n-1} \omega_{n j} A_{h} U^{j}=k \sum_{l=1}^{M} \gamma_{l} e^{-v_{l} t_{n}} S_{l}^{n}, \quad \text { where } S_{l}^{n}=\sum_{j=1}^{n-1} e^{v_{l} t_{j}} A_{h} U^{j} .
$$

The $U^{j}$ now only enter in the subsequent calculations through the $S_{l}^{n}$ and may therefore be discarded once the $S_{l}^{n}$ have been updated.

We now turn to the case of a kernel $\beta(t)$ that is weakly singular at $t=0$. In this case the integrand is singular even when the solution is smooth, and we shall therefore use product integration.

We shall first consider the quadrature rule obtained by approximating $\varphi$ in the integrand by a piecewise constant function $\bar{\varphi}(t)$ taking the value $\varphi\left(t_{j}\right)$ in $\left(t_{j-1}, t_{j}\right]$, and thus use

$$
q_{n}(\varphi)=\sum_{j=1}^{n} \int_{t_{j-1}}^{t_{j}} \beta\left(t_{n}-s\right) \varphi\left(t_{j}\right) d s=\sum_{j=1}^{n} \kappa_{n-j} \varphi\left(t_{j}\right),
$$

where

$$
\kappa_{j}=\int_{t_{j}}^{t_{j+1}} \beta(s) d s .
$$

For $\beta$ convex on $\mathbf{R}_{+}$we shall show the following: 
LEMMA 4.7. Assume that $\beta \in L_{1}(0, T)$ and satisfies (4.4). Then the integration rule (4.13), (4.14) is positive and we have

$$
\tilde{\epsilon}_{N}(\varphi) \leq C_{T} k \int_{0}^{t_{N}}\left\|\varphi_{i}\right\| d s, \quad \text { for } t_{N} \leq T .
$$

Proof. The sequence $\left\{\kappa_{j}\right\}_{j=0}^{\infty}$ is convex since, as a result of the convexity of $\beta(t)$,

$$
\kappa_{j+2}-2 \kappa_{j+1}+\kappa_{j}=\int_{t_{j}}^{t_{j+1}}\{\beta(s+2 k)-2 \beta(s+k)+\beta(s)\} d s \geq 0 .
$$

The $\kappa_{j}$ are also bounded, so we conclude from Lemma 4.3, as in the case of a smooth $\beta$, that the quadrature formula is positive.

Next, by the definition of the $\kappa_{j}$ we have

$$
\epsilon_{n}(\varphi)=\sum_{j=1}^{n} \int_{t_{j-1}}^{t_{j}} \beta\left(t_{n}-s\right)\left\{\varphi\left(t_{j}\right)-\varphi(s)\right\} d s,
$$

so that

$$
\left|\epsilon_{n}(\varphi)\right| \leq \sum_{j=1}^{n} \int_{t_{j-1}}^{t_{j}} \beta\left(t_{n}-s\right) \int_{t_{j-1}}^{t_{j}}\left|\varphi^{\prime}(\sigma)\right| d \sigma d s \leq \sum_{j=1}^{n} \kappa_{n-j} \int_{t_{j-1}}^{t_{j}}\left|\varphi^{\prime}(\sigma)\right| d \sigma .
$$

Applying Minkowski's inequality, summing over $n$ and then reversing the order of summation, we arrive at

$$
k \sum_{n=1}^{N}\left\|\epsilon_{n}(\varphi)\right\| \leq k \sum_{j=1}^{N}\left\{\sum_{n=j}^{N} \kappa_{n-j}\right\} \int_{t_{j-1}}^{t_{j}}\left\|\varphi_{t}\right\| d s,
$$

whence the result, because

$$
\sum_{n=j}^{N} \kappa_{n-j}=\int_{0}^{t_{N-j+1}} \beta(s) d s \leq\|\beta\|_{L_{1}(0, T)}, \quad \text { for } t_{N} \leq T .
$$

For the special case $\beta(t)=t^{-1 / 2} / \Gamma(1 / 2)=(\pi t)^{-1 / 2}$, using an argument involving generating functions, Sanz-Serna [16] has proposed the quadrature rule

$$
q_{n}(\varphi)=k^{1 / 2} \sum_{j=1}^{n} \gamma_{n-j} \varphi^{j}, \quad \text { where } \gamma_{j}=(-1)^{j}\left(\begin{array}{c}
-1 / 2 \\
j
\end{array}\right)=\frac{(2 j-1) ! !}{(2 j) ! !}
$$


and proved an error estimate for the homogeneous equation by spectral methods. His analysis has recently been carried further by Lopez-Marcos [11] using arguments of the type of the present paper. We shall demonstrate that this rule fits into the present framework.

It is easy to see that $\left\{\gamma_{j}\right\}_{j=0}^{\infty}$ is convex and that $\gamma_{j} \rightarrow 0$ as $j \rightarrow \infty$, so that Lemma 4.3 applies and hence the quadrature formula is positive. In order to show that it is first-order accurate and to derive an estimate for the global quadrature error, we note that since this has already been shown for our scheme (4.13), (4.14), it suffices to study the difference between the two quadrature formulas, i.e.,

$$
d_{n}(\varphi)=k^{1 / 2} \sum_{j=1}^{n} \delta_{n-j} \varphi\left(t_{j}\right), \quad \text { where } \delta_{j}=\gamma_{j}-k^{-1 / 2} \kappa_{j} .
$$

We shall show that, uniformly in $\Omega$,

$$
\left|d_{n}(\varphi)\right| \leq C k\left\{t_{n}^{-1 / 2}|\varphi(0)|+\sum_{j=1}^{n} t_{n+1-j}^{-1 / 2} \int_{t_{j-1}}^{t_{j}}\left|\varphi^{\prime}(s)\right| d s\right\}, \quad \text { for } n \geq 1,
$$

from which it follows easily that

$$
\tilde{\epsilon}_{N}(\varphi) \leq C k\left\{\|\varphi(0)\|+\int_{0}^{t_{N}}\left\|\varphi_{t}\right\| d s\right\}, \quad \text { for } t_{n} \leq T .
$$

In conjunction with Theorem 3.1 and given sufficient regularity, this yields an $O\left(h^{r}+k\right)$ error estimate, which slightly improves the result in [16].

It remains to show (4.15). For this purpose, we set $\sigma_{j}=\sum_{l=0}^{j} \delta_{l}$, for $j \geq 0$, with $\sigma_{-1}=0$, and note that by summation by parts,

$$
d_{n}(\varphi)=k^{1 / 2}\left\{\sigma_{n-1} \varphi(0)+\sum_{j=1}^{n} \sigma_{n-j}\left(\varphi\left(t_{j}\right)-\varphi\left(t_{j-1}\right)\right)\right\} .
$$

The desired result therefore at once follows from $\left|\sigma_{n}\right| \leq C(n+1)^{-1 / 2}$, for $n \geq 0$. To prove this bound for $\sigma_{n}$ we note that by (4.14)

$k^{-1 / 2} \sum_{j=0}^{n} \kappa_{j}=k^{-1 / 2} \int_{0}^{t_{n+1}} \beta(s) d s=(k \pi)^{-1 / 2} \int_{0}^{t_{n+1}} s^{-1 / 2} d s=2 \pi^{-1 / 2}(n+1)^{1 / 2}$.

Further, using, as in [16], a known identity for binomial coefficients and Stirling's formula, we have

$$
\sum_{j=0}^{n} \gamma_{j}=2(n+1) \gamma_{n+1}=2(n+1)^{1 / 2} \pi^{-1 / 2}+O\left((n+1)^{-1 / 2}\right) .
$$


Together these estimates show

$$
\left|\sigma_{n}\right|=\left|\sum_{j=0}^{n} \gamma_{j}-k^{-1 / 2} \sum_{j=0}^{n} \kappa_{j}\right| \leq C(n+1)^{-1 / 2},
$$

which completes the proof.

We shall complete this section by exhibiting a second-order quadrature rule which is weakly positive for any positive-definite kernel $\beta$, not just for kernels satisfying (4.4). To do so, we replace $\varphi$ in the integrand by its continuous piecewise linear interpolant, i.e., we set

$$
\begin{aligned}
q_{n}(\varphi) & =\sum_{j=1}^{n} k^{-1} \int_{t_{j-1}}^{t_{j}} \beta\left(t_{n}-s\right)\left\{\left(t_{j}-s\right) \varphi\left(t_{j-1}\right)+\left(s-t_{j-1}\right) \varphi\left(t_{j}\right)\right\} d s \\
& =\sum_{j=0}^{n} \tilde{\kappa}_{n j} \varphi\left(t_{j}\right)
\end{aligned}
$$

where, in terms of the hat function $h(t)=\max (1-|t|, 0)$,

$$
\tilde{\kappa}_{n j}=\int_{-\min \left(k, t_{j}\right)}^{\min \left(k, t_{n-j}\right)} \beta\left(t_{n-j}-s\right) h(s / k) d s .
$$

LEMMA 4.8. Assume that $\beta \in L_{1}(0, T)$ is a positive-definite kernel. Then the integration rule (4.16) is weakly positive, and satisfies

$$
\tilde{\epsilon}_{N}(\varphi) \leq C k \int_{0}^{k}\left\|\varphi_{t}\right\| d s+C k^{2} \int_{k}^{t_{N}}\left\|\varphi_{t}\right\| d s, \quad \text { for } t_{N} \leq T .
$$

PROOF. Recalling the notation $\bar{\varphi}(t)$ for the piecewise constant function taking the value $\Phi^{j}=\varphi\left(t_{j}\right)$ in $\left(t_{j-1}, t_{j}\right]$, we may write

$$
\bar{\varphi}(t)=\sum_{j=1}^{N} \Phi^{j} \chi_{j}(t), \quad \text { in }\left(0, t_{N}\right),
$$

where $\chi_{j}$ is the characteristic function of $\left(t_{j-1}, t_{j}\right]$. Writing

$$
\int_{0}^{t_{N}} \int_{0}^{t} \beta(t-s) \bar{\varphi}(s) \bar{\varphi}(t) d s d t=\sum_{n=1}^{N} \sum_{j=1}^{n} b_{n j} \Phi^{j} \Phi^{n},
$$

a simple calculation shows that, with $\tilde{\kappa}_{n j}$ defined in (4.17),

$$
b_{n j}=\int_{t_{n-1}}^{t_{n}} \int_{t_{j-1}}^{\min \left(t_{j}, t\right)} \beta(t-s) d s d t=k \tilde{\kappa}_{n j},
$$


and thus, since $\beta$ is positive definite,

$$
Q_{N}(\Phi)=\int_{0}^{t_{N}} \int_{0}^{t} \beta(t-s) \bar{\varphi}(s) d s \bar{\varphi}(t) d t \geq 0, \quad \text { when } \Phi^{0}=0 .
$$

We now consider the accuracy and global quadrature error of $q_{n}$. Using Newton's form of the remainder for linear interpolation, we find that

$$
\epsilon_{n}(\varphi)=\sum_{j=1}^{n} \int_{t_{j-1}}^{t_{j}} \beta\left(t_{n}-s\right)\left(s-t_{j-1}\right)\left(t_{j}-s\right) \varphi\left[t_{j-1}, t_{j}, s\right] d s .
$$

In the first term, we use the estimate

$$
|s \varphi[0, k, s]|=|\varphi[s, k]-\varphi[0, k]| \leq 2 \int_{0}^{k}\left|\varphi^{\prime}(y)\right| d y, \quad \text { for } 0<s<k,
$$

and in the remaining terms we apply, with $K_{j}$ the Peano kernel for the divided difference,

$$
\begin{aligned}
\left|\varphi\left[t_{j-1}, t_{j}, s\right]\right| & =\left|\int_{t_{j-1}}^{t_{j}} K_{j}(s, y) \varphi^{\prime \prime}(y) d y\right| \\
& \leq k^{-1} \int_{t_{j-1}}^{t_{j}}\left|\varphi^{\prime \prime}(y)\right| d y, \quad \text { for } t_{j-1}<s<t_{j} .
\end{aligned}
$$

Hence, with $\mu_{j}=\int_{t_{j}}^{t_{j+1}}|\beta(s)| d s$, we have

$$
\left|\epsilon_{n}(\varphi)\right| \leq 2 k \mu_{n-1} \int_{0}^{k}\left|\varphi^{\prime}(y)\right| d y+k^{2} \sum_{j=2}^{n} \mu_{n-j} \int_{t_{j-1}}^{t_{j}}\left|\varphi^{\prime \prime}(y)\right| d y,
$$

from which our statement follows as in the proof of Lemma 4.7.

\section{Regularity of solutions}

In this section we shall present some results concerning the regularity of solutions of our integro-differential equations. The purpose is to exhibit conditions on the data which are sufficient for our error estimates in Section 3 to be applicable. We first define our solution concept by means of a representation formula 
in terms of data, and show the existence of a unique such solution, requiring only that $\beta \in L_{1, \text { loc }}\left(\overline{\mathbf{R}}_{+}\right)$. We then show regularity results under various assumptions on $\beta$ and the data. In the latter part of the section we study the particular weakly singular case $\beta(t)=t^{\alpha-1} / \Gamma(\alpha), 0<\alpha<1$. For the case of a smooth kernel $\beta$, any regularity desired can be guaranteed under the appropriate assumptions on data, whereas in the weakly singular case the regularity is limited.

Letting * denote Laplace convolution, so that

$$
(f * g)(t)=\int_{0}^{t} f(t-s) g(s) d s, \quad t \geq 0,
$$

and using a dash to denote differentiation with respect to time, the initial-value problem (1.1) may be written as

$$
u^{\prime}+\beta * A u=f, \quad \text { for } t \geq 0, u(0)=v,
$$

where we also keep in mind that $u(t)=0$ on $\partial \Omega$ for all $t>0$. We begin by using a spectral decomposition to derive a formal representation of the solution of this problem. Letting $\left\{\lambda_{j}\right\}_{j=1}^{\infty}$ and $\left\{\varphi_{j}\right\}_{j=1}^{\infty}$ denote the eigenvalues and normalised eigenfunctions of $A$, we find for the Fourier coefficients $u_{j}(t)=\left(u(t), \varphi_{j}\right)$ of the solution that

$$
u_{j}^{\prime}+\lambda_{j} \beta * u_{j}=f_{j}, \quad \text { for } t \geq 0, u_{j}(0)=v_{j},
$$

where $f_{j}=f_{j}(t)$ and $v_{j}$ are the Fourier coefficients of $f$ and $v$. For $\lambda>0$, suppose that $W_{\lambda}$ satisfies

$$
W_{\lambda}^{\prime}+\lambda \beta * W_{\lambda}=0, \quad \text { for } t \geq 0, \quad W_{\lambda}(0)=1 .
$$

It is then easy to see that a solution of (5.2) is provided by

$$
u_{j}=W_{\lambda_{j}} v_{j}+W_{\lambda_{j}} * f_{j}
$$

Thus, a formal solution of (5.1) is given by

$$
u(t)=E(t) v+\int_{0}^{t} E(t-s) f(s) d s, \quad \text { for } t \geq 0,
$$

where the linear operator $E(t)$ is defined by

$$
E(t) v=\sum_{j=1}^{\infty} W_{\lambda_{j}}(t)\left(v, \varphi_{j}\right) \varphi_{j}
$$


Note that each $\varphi_{j}$ vanishes on $\partial \Omega$, so the function $u$ defined by (5.4) satisfies the boundary conditions, at least formally.

We now show that (5.3) does indeed have a solution such that the operator $E(t)$ is uniformly bounded in $L_{2}(\Omega)$.

THEOREM 5.1. Assume that $\beta \in L_{1}(0, T)$ for each $T>0$ and that $\beta$ is positive definite. Then, for each $\lambda \geq 0$, the initial value problem (5.3) has a unique solution in $C^{1}\left(\overline{\mathbf{R}}_{+}\right)$, and

$$
\left|W_{\lambda}(t)\right| \leq 1, \quad \text { for } t \geq 0 .
$$

Further, the sum (5.5) defines a bounded mapping in $L_{2}(\Omega)$ with

$$
\|E(t) v\| \leq\|v\|, \quad \text { for } t \geq 0 \text { and } v \in L_{2}(\Omega) .
$$

Moreover, for each $v \in L_{2}(\Omega)$, the mapping $t \mapsto E(t) v$ is continuous from $\overline{\mathbf{R}}_{+}$ into $L_{2}(\Omega)$.

PROOF. With $\tilde{\beta}(t)=\int_{0}^{t} \beta(s) d s,(5.3)$ is equivalent to the Volterra equation

$$
W_{\lambda}(t)+\lambda \int_{0}^{t} \tilde{\beta}(t-s) W_{\lambda}(s) d s=1, \quad \text { for } t \geq 0,
$$

which has a unique solution in $C^{1}\left(\overline{\mathbf{R}}_{+}\right)$because $\tilde{\beta}$ is continuous on $\overline{\mathbf{R}}_{+}$. To obtain the estimate for $W_{\lambda}(t)$, we multiply both sides of (5.3) by $2 W_{\lambda}$, then integrate over $[0, T]$ and use the positive definiteness of $\beta$ to conclude that $W_{\lambda}(T)^{2}-W_{\lambda}(0)^{2} \leq 0$. Parseval's relation now implies (5.6) and also that, for a fixed $v \in L_{2}(\Omega)$, the sum (5.5) is continuous for $t \in \overline{\mathbf{R}}_{+}$.

For $v \in L_{2}(\Omega)$ and $f \in C\left([0, T] ; L_{2}(\Omega)\right)$, and for each $T>0$, the representation formula (5.4) defines a function $u \in C\left([0, T] ; L_{2}(\Omega)\right)$, which we shall thus consider our solution of (5.1), and for which, by (5.6),

$$
\|u(t)\| \leq\|v\|+\int_{0}^{t}\|f(s)\| d s \quad \text { for } 0 \leq t \leq T,
$$

cf. (1.4). We proceed to discuss conditions on the data $v$ and $f$ for this solution to possess various regularity properties. In particular, these will contain conditions for the solution to belong to $C^{1}\left([0, T], L_{2}(\Omega)\right) \cap C([0, T], D(A))$, and thus to be a genuine solution of $(5.1)$ on $[0, T]$. 
Given $r \in \mathbf{R}$, let $\dot{H}^{r}(\Omega)$ denote the subspace of $L_{2}(\Omega)$ consisting of those functions $v$ for which the norm

$$
|v|_{r}=\left(\sum_{j=1}^{\infty} \lambda_{j}^{r}\left(v, \varphi_{j}\right)^{2}\right)^{1 / 2}
$$

is finite. It can be shown that for $r \geq 0$ (and $r-1 / 2$ not an integer) $v \in \dot{H}^{r}$ if and only if $v \in H^{r}(\Omega)$ and $A^{j} v=0$ on $\partial \Omega$ for all integers $j$ with $0 \leq j<r / 2$. Moreover, the norm (5.7) is then equivalent to the usual norm in $H^{r}(\Omega)$, which we have been writing simply as $\|v\|_{r}$ (cf. $[8,19]$ ).

In discussing the regularity of $u$, it is convenient to deal separately with each of the two terms in (5.4). We consider first the homogeneous equation and denote by $[y]$ the largest integer $\leq y$.

THEOREM 5.2. Let $m \geq 0$ and suppose $\beta^{(p)} \in L_{1}(0, T)$ for some $T>0$, with $p=\max \{0, m-1\}$. Then

$$
\left|E^{(m)}(t) v\right|_{r} \leq C|v|_{r+2[(m+1) / 2]}, \quad \text { for } 0 \leq t \leq T, r \geq 0,
$$

where $C$ depends on $\beta, T, m$, and $\lambda_{1}$.

ProOF. In view of (5.5) it suffices to show that $W_{\lambda} \in C^{m}([0, T])$ and

$$
\left|W_{\lambda}^{(m)}(t)\right| \leq C \lambda^{[(m+1) / 2]} \quad \text { for } 0 \leq t \leq T \text { and } \lambda \geq \lambda_{1} .
$$

In the process of doing so we also show that

$$
\left|W_{\lambda}^{(m)}(0)\right| \leq C \lambda^{[m / 2]}, \quad \text { for } \lambda \geq \lambda_{1} .
$$

These estimates will be shown by induction on $m$. Since they are clear for $m=0$ by Theorem 5.1 , we now assume $m \geq 1$ and that they have been shown up to $m-1$. Differentiating (5.3) $m-1$ times we have

$$
W_{\lambda}^{(m)}+\lambda \beta * W_{\lambda}^{(m-1)}=-\lambda \sum_{l=0}^{m-2} W_{\lambda}^{(l)}(0) \beta^{(m-2-l)} \equiv b_{m-1}(\lambda, t) .
$$

By our induction hypothesis, we see first that $W^{(m)} \in C([0, T])$ and

$$
\left|W_{\lambda}^{(m)}(0)\right|=\left|b_{m-1}(\lambda, 0)\right| \leq C \lambda^{1+[(m-2) / 2]}=C \lambda^{[m / 2]}, \quad \text { for } \lambda \geq \lambda_{1} .
$$


Replacing $m$ by $m+1$ in (5.8) gives an equation of the form (5.2) for $W_{\lambda}^{(m)}$, and therefore

$$
W_{\lambda}^{(m)}(t)=W_{\lambda}(t) W_{\lambda}^{(m)}(0)+\int_{0}^{t} W_{\lambda}(t-s) b_{m}(\lambda, s) d s
$$

It follows that

$$
\left|W_{\lambda}^{(m)}(t)\right| \leq\left|W_{\lambda}^{(m)}(0)\right|+C \lambda \sum_{l=0}^{m-1}\left|W_{\lambda}^{(l)}(0)\right| \leq C \lambda^{[(m+1) / 2]},
$$

and the induction goes through.

We now turn to the nonhomogeneous equation with zero initial data, and begin to consider the regularity in time.

THEOREM 5.3. Let $m \geq 0$ and assume $\beta^{(p)} \in L_{1}(0, T)$, where $p=\max \{0, m-2\}$. Then, if $v=0$, we have for the solution (5.4)

$$
\left\|u^{(m)}(t)\right\| \leq C \sum_{l=0}^{m-1}\left|f^{(l)}(0)\right|_{2[(m-l) / 2]}+\int_{0}^{t}\left\|f^{(m)}(s)\right\| d s, \quad \text { for } t \in[0, T] .
$$

Proof. Putting $v=0$ in (5.4) and differentiating $m$ times, we get

$$
u^{(m)}(t)=\sum_{l=0}^{m-1} E^{(m-1-l)}(t) f^{(l)}(0)+\int_{0}^{t} E(t-s) f^{(m)}(s) d s,
$$

from which the result follows by Theorem 5.2.

In order to discuss regularity in space, it is convenient to introduce the notation

$$
\|f\|_{m, r}=\|f\|_{C^{m}\left([0, T) ; H^{r}(\Omega)\right)}=\max _{0 \leq l \leq m} \max _{0 \leq t \leq T}\left\|f^{(l)}(t)\right\|_{r}
$$

for the $C^{m}$-norm of a function $f:[0, T] \rightarrow H^{r}(\Omega)$, where, for brevity, we suppress the dependence on $T$ in the notation. 
THEOREM 5.4. Let $m \geq 0$ and $r \geq 1$ be integers and assume $\beta^{(m+2 r-1)} \in$ $L_{1}(0, T)$. If $v=0$, then for the solution of $(5.1)$ we have

$$
\begin{aligned}
\|u\|_{m, 2 r} \leq C\left(\sum_{l=0}^{m+2 r-2}\left|f^{(l)}(0)\right|_{2[(m+2 r-l) / 2]}\right. & \\
& \left.+\sum_{l=1}^{r}\|f\|_{m+2 l-1,2 r-2 l}+\int_{0}^{T}\left\|f^{(m+2 r)}\right\| d s\right) .
\end{aligned}
$$

PROOF. We differentiate (5.1) to obtain

$$
\beta(0) A u+\beta^{\prime} * A u=f^{\prime}-u^{\prime \prime},
$$

and think of this as a Volterra equation for $A u$. Since $\beta$ is positive definite it is easy to see that $\beta(0)>0$ (unless $\beta \equiv 0$ ). Let $\gamma$ be the resolvent convolution kernel for (5.10), satisfying

$$
\beta(0) \gamma+\beta^{\prime} * \gamma=\beta^{\prime}, \quad \text { for } t \in[0, T] .
$$

Then $\gamma^{(m+2 r-2)} \in L_{1}(0, T)$ and we have

$$
\beta(0) A u=\left(f^{\prime}-u^{\prime \prime}\right)-\gamma *\left(f^{\prime}-u^{\prime \prime}\right) .
$$

Since $u=0$ on $\partial \Omega$, we conclude from elliptic regularity theory [9] that, for $0 \leq l \leq r-1$,

$$
\begin{aligned}
\|u\|_{m+2 l, 2 r-2 l} & \leq C\left\|f^{\prime}-u^{\prime \prime}\right\|_{m+2 l, 2 r-2 l-2} \\
& \leq C\left(\|f\|_{m+2 l+1,2 r-2 l-2}+\|u\|_{m+2 l+2,2 r-2 l-2}\right),
\end{aligned}
$$

whence, by repeated application,

$$
\|u\|_{m, 2 r} \leq C\left(\sum_{l=1}^{r}\|f\|_{m+2 l-1,2 r-2 l}+\|u\|_{m+2 r, 0}\right) .
$$

The proof is now completed by using Theorem 5.3 to bound the last term.

As an example, assume $\beta \in C^{1}([0, T])$ and consider the backward Euler method (3.2) using the right-hand rectangle rule (4.5). Theorem 3.1 (with $r=2$ ) and Lemma 4.4 together imply the error estimate

$$
\left\|U^{n}-u\left(t_{n}\right)\right\| \leq C\left(h^{2}+k\right)\left\{\|v\|_{2}+\int_{0}^{t_{n}}\left(\left\|u_{t t}\right\|+\left\|u_{t}\right\|_{2}\right) d s\right\}, \quad \text { for } t_{n} \leq T,
$$


and, provided $\beta^{\prime \prime} \in L_{1}(0, T)$, our regularity results in Theorems 5.2-5.4 give

$$
\begin{aligned}
\|v\|_{2}+\int_{0}^{T}\left(\left\|u_{t t}\right\|\right. & \left.+\left\|u_{t}\right\|_{2}\right) d s \\
& \leq C\left\{|v|_{4}+|f(0)|_{2}+\left|f^{\prime}(0)\right|_{2}+\|f\|_{2,0}+\int_{0}^{T}\left\|f^{\prime \prime \prime}(s)\right\| d s\right\} .
\end{aligned}
$$

Of course, the latter estimate is meaningful only if the right-hand side is finite, and this requires the validity of the conditions

$$
v=A v=f(0)=f^{\prime}(0)=0 \quad \text { on } \partial \Omega,
$$

partly caused by our considering the two terms in (5.4) separately.

For the remainder of this section, in order to consider an example of a weaklysingular kernel, we shall consider in some detail the kernel $\beta(t)=\omega_{\alpha}(t)$, with $0<\alpha<1$, where

$$
\omega_{\rho}(t)=t^{\rho-1} / \Gamma(\rho), \quad \text { for } t>0, \rho>0 .
$$

The kernel $\omega_{\alpha}, 0<\alpha<1$, is positive definite because it satisfies (4.4). However, since $\omega_{\alpha}^{\prime} \notin L_{1}(0, T)$, Theorems 5.2 and 5.3 apply only when $m=1$, while Theorem 5.4 does not apply at all. We shall see that, even for the special case of the homogeneous equation with a fixed eigenfunction $\varphi_{j}$ as initial data, we have $u^{(m)}(t) \sim c_{m} t^{\alpha+1-m}$ for small $t$, so that, in particular, $u_{t t}$ is weakly singular and $u_{t t t} \notin L_{1}(0, T)$.

The convolution operator $f \mapsto \omega_{\rho} * f$, with $\omega_{\rho}$ defined in (5.13), is known as the Riemann-Liouville fractional integral of order $\rho$ (see [1, page 393] for a detailed discussion). By computing the Laplace transform

$$
\hat{\omega}_{\rho}(\sigma)=\frac{1}{\Gamma(\rho)} \int_{0}^{\infty} e^{-\sigma t} t^{\rho-1} d t=\sigma^{-\rho}, \quad-\pi / 2 \leq \arg \sigma \leq \pi / 2,
$$

we see at once that

$$
\omega_{\rho_{1}} * \omega_{\rho_{2}}=\omega_{\rho_{1}+\rho_{2}}, \quad \text { for } \rho_{1}>0 \text { and } \rho_{2}>0,
$$

a property we will now use to obtain an explicit representation for $W_{\lambda}$.

LEMMA 5.1. In the case of the weakly singular kernel $\beta=\omega_{\alpha}$, the solution of (5.3) is given by

$$
W_{\lambda}(t)=W\left(\lambda^{1 /(1+\alpha)} t\right), \quad \text { for } t \geq 0 \text { and } \lambda \geq 0,
$$


where the function $W$ is defined by the series

$$
W(t)=\sum_{n=0}^{\infty}(-1)^{n} \frac{t^{n(1+\alpha)}}{\Gamma[1+n(1+\alpha)]}=\sum_{n=0}^{\infty}(-1)^{n} \omega_{1+n(1+\alpha)}(t), \quad \text { for } t \geq 0 .
$$

PROOF. Since $\omega_{1+\rho}^{\prime}=\omega_{\rho}$ for $\rho>0$, and $\omega_{1}=1$, it follows using (5.15) that

$$
W^{\prime}=\sum_{n=1}^{\infty}(-1)^{n} \omega_{n(1+\alpha)}=\sum_{n=0}^{\infty}(-1)^{n+1} \omega_{\alpha} * \omega_{1+n(1+\alpha)}=-\beta * W,
$$

whence, since $W(0)=1, W$ is the solution of (5.3) in the special case $\lambda=1$. Therefore, the function defined by (5.16) satisfies $W_{\lambda}(0)=1$ and, with $v=$ $\lambda^{1 /(1+\alpha)}$,

$$
\begin{aligned}
W_{\lambda}^{\prime}(t) & =v W^{\prime}(v t)=-v(\beta * W)(v t)=-v \int_{0}^{v t} \beta(v t-s) W(s) d s \\
& =-v^{2} \int_{0}^{t} \beta[v(t-s)] W(v s) d s=-v^{1+\alpha} \int_{0}^{t} \beta(t-s) W_{\lambda}(s) d s \\
& =-\lambda\left(\beta * W_{\lambda}\right)(t),
\end{aligned}
$$

as claimed.

We observe in passing that $W(t)=e^{-t}$ in the limiting case $\alpha=0$, and that $W(t)=\cos t$ in the other limiting case $\alpha=1$.

In particular, Lemma 5.1 shows that $W^{(m)}(t) \sim c_{m} t^{\alpha+1-m}$ for small $t$, which implies our earlier statement about the behaviour of a solution of the homogeneous equation corresponding to a simple initial Fourier mode. In order to derive more complete error estimates we shall have use also for information concerning the behaviour of $W(t)$ for large $t$.

LEMMA 5.2. For $0<\alpha<1$, the function $W$ defined in (5.17) has the asymptotic expansion

$$
W(t) \sim-\frac{1}{\pi} \sum_{n=1}^{\infty} \sin (\pi n \alpha) \Gamma[n(\alpha+1)] t^{-n(\alpha+1)}, \quad \text { as } t \rightarrow \infty
$$


PROOF. Using (5.14) to take Laplace transforms in (5.3) with $\lambda=1$, we find

$$
\widehat{W}(\sigma)=\frac{\sigma^{\alpha}}{1+\sigma^{\alpha+1}}, \quad-\pi / 2 \leq \arg \sigma \leq \pi / 2 .
$$

Therefore, the inversion formula yields the integral representation

$$
W(t)=\frac{1}{2 \pi i} \int_{\operatorname{Re}(\sigma)=\omega} \frac{e^{\sigma t} \sigma^{\alpha}}{1+\sigma^{\alpha+1}} d \sigma, \quad \text { for } t>0, \omega>0 .
$$

For any complex number $z$, let $(-\infty, z]$ denote the set of complex numbers $\sigma$ statisfying $-\infty<\operatorname{Re}(\sigma) \leq \operatorname{Re}(z)$ and $\operatorname{Im}(\sigma)=\operatorname{Im}(z)$. Next, for $\epsilon>0$, let $\mathcal{C}_{\epsilon}(z)$ denote the contour formed by the lines $(-\infty, z \pm i \epsilon]$ and the semicircle $\sigma=z+\epsilon e^{i \theta}(-\pi / 2<\theta<\pi / 2)$, oriented so that $\mathcal{C}_{\epsilon}(z)$ passes around $z$ counterclockwise. The integrand in (5.18) has branch points at $\sigma=0$ and $\sigma=e^{ \pm i \pi /(\alpha+1)}$, so we cut the complex plane along the negative real axis $(-\infty, 0]$ and along the lines $\left(-\infty, e^{ \pm i \pi /(\alpha+1)}\right]$, and write

$$
\frac{1}{2 \pi i} \int_{\operatorname{Re}(\sigma)=\omega} \frac{e^{\sigma t} \sigma^{\alpha}}{1+\sigma^{\alpha+1}} d \sigma=\frac{1}{2 \pi i} \int_{\mathcal{C}_{\epsilon}(0)} \frac{e^{\sigma t} \sigma^{\alpha}}{1+\sigma^{\alpha+1}} d \sigma+E_{+}(t)+E_{-}(t),
$$

where

$$
E_{ \pm}(t)=\frac{1}{2 \pi i} \int_{\mathcal{C}_{\epsilon}\left(e^{ \pm i \pi /(\alpha+1)}\right)} \frac{e^{\sigma t} \sigma^{\alpha}}{1+\sigma^{\alpha+1}} d \sigma .
$$

Since $\operatorname{Re}\left(e^{ \pm i \pi /(\alpha+1)}\right)<0$, it is clear that $E_{ \pm}(t)$ is exponentially small as $t \rightarrow \infty$, and since

$$
\frac{1}{1+\sigma^{\alpha+1}}=\sum_{n=0}^{N}(-1)^{n} \sigma^{n(\alpha+1)}+\frac{(-1)^{N+1} \sigma^{(N+1)(\alpha+1)}}{1+\sigma^{\alpha+1}},
$$

we see that

$$
\frac{1}{2 \pi i} \int_{\mathcal{C}_{\epsilon}(0)} \frac{e^{\sigma t} \sigma^{\alpha}}{1+\sigma^{\alpha+1}} d \sigma=\sum_{n=0}^{N} \frac{(-1)^{n}}{2 \pi i} \int_{\mathcal{C}_{\epsilon}(0)} e^{\sigma t} \sigma^{(n+1)(\alpha+1)-1} d \sigma+E_{N}(t),
$$

where

$$
E_{N}(t)=\frac{(-1)^{N+1}}{2 \pi i} \int_{\mathcal{C}_{\epsilon}(0)} \frac{e^{\sigma t} \sigma^{(N+2)(\alpha+1)-1}}{1+\sigma^{\alpha+1}} d \sigma, \quad \text { for } t>0 .
$$


Finally,

$$
\begin{aligned}
\lim _{\epsilon \downarrow 0} \frac{(-1)^{n}}{2 \pi i} \int_{\mathcal{C}_{\epsilon}(0)} e^{\sigma t} \sigma^{(n+1)(\alpha+1)-1} d \sigma \\
=-\frac{1}{\pi} \sin [\pi(n+1) \alpha] \Gamma[(n+1)(\alpha+1)] t^{-(n+1)(\alpha+1)}
\end{aligned}
$$

and

$$
\left|E_{N}(t)\right| \leq \frac{1}{\pi} \int_{0}^{\infty} \frac{e^{-x t} x^{(N+2)(\alpha+1)-1}}{\sin ^{2}(\pi \alpha)} d x=\frac{\Gamma[(N+2)(\alpha+1)]}{\pi \sin ^{2}(\pi \alpha)} t^{-(N+2)(\alpha+1)},
$$

giving the asymptotic expansion as claimed.

Using the functional identity $\Gamma(z) \Gamma(1-z)=\pi / \sin (\pi z)$, we can rewrite the expansion in the form

$$
W(t) \sim-\sum_{n=1}^{\infty} \frac{(-1)^{n} t^{-n(\alpha+1)}}{\Gamma[1-n(\alpha+1)]} \quad \text { as } t \rightarrow \infty,
$$

which compares with (5.17).

We are now ready to state and prove our regularity results for (5.1) with the weakly singular kernel $\omega_{\alpha}$. We begin with the homogeneous equation.

THEOREM 5.5. Let $\beta=\omega_{\alpha}$, with $0<\alpha<1$, and let $r \in \mathbf{R}$. Then

$$
|E(t) v|_{r+2 \mu} \leq C t^{-(\alpha+1) \mu}|v|_{r}, \quad \text { for } t>0 \text { and } 0 \leq \mu \leq 1,
$$

with $C$ depending on $\alpha$. When $m \geq 1$ we have

$$
\left|E^{(m)}(t) v\right|_{r+2 \mu} \leq C t^{-(\alpha+1) \mu-m}|v|_{r}, \quad \text { for } t>0 \text { and }-1 \leq \mu \leq 1 \text {, }
$$

where $C$ depends on $\alpha$ and $m$.

PROOF. By (5.17) and Lemma 5.2 we have

$$
|W(t)| \leq C \min \left(1, t^{-(\alpha+1)}\right), \quad \text { for } t>0,
$$

and hence, using (5.16),

$$
\left|W_{\lambda}(t)\right| \leq C t^{-(\alpha+1) \mu} \lambda^{-\mu}, \quad \text { for } 0 \leq \mu \leq 1 \text { and } \lambda>0,
$$


which shows the first estimate of the theorem. To show the second we find similarly that since $W^{(m)}(t)=O\left(t^{\alpha+1-m}\right)$ for small $t$, and $W^{(m)}(t)=O\left(t^{-\alpha-1-m}\right)$ for large $t$, we have

$$
\left|W^{(m)}(t)\right| \leq C t^{-(\alpha+1) \mu-m}, \quad \text { for }|\mu| \leq 1,
$$

and hence by (5.16)

$$
\left|W_{\lambda}^{(m)}(t)\right| \leq C t^{-(\alpha+1) \mu-m} \lambda^{-\mu}, \quad \text { for }|\mu| \leq 1 \text { and } \lambda>0 .
$$

The solution of the homogeneous equation is thus smoother than the initial data by two derivatives, for $t>0$, with a bound which deteriorates faster as $t \rightarrow 0$ the weaker the singularity. As $\alpha \rightarrow 0$ we recognise formally the smoothing property of the parabolic equation. We also note that although the smoothing with respect to $x$ is limited to two derivatives, the solution is infinitely differentiable with respect to time. Here, the behaviour of the bound in (5.19) may be chosen less singular than $t^{-m}$ by sacrificing some of the spatial regularity. This is useful in the following result which concerns the nonhomogeneous equation with vanishing initial data. Here we concentrate on results which are of interest for our numerical error estimates.

THEOREM 5.6. Let $\beta=\omega_{\alpha}$, with $0<\alpha<1$, and let $m \geq 1$ and $T>0$. If $\left|\mu_{l}\right| \leq 1$ for $0 \leq l \leq m-2$, then we have for the solution of (5.1), with $v=0$,

$$
\begin{aligned}
\left\|u^{(m)}(t)\right\| \leq C \sum_{l=0}^{m-2} t^{\mu_{l}(\alpha+1)-(m-1-l)}\left|f^{(l)}(0)\right|_{2 \mu_{l}} & \\
& +\left\|f^{(m-1)}(0)\right\|+\int_{0}^{t}\left\|f^{(m)}\right\| d s, \quad t \in(0, T] .
\end{aligned}
$$

Further, if $0<\epsilon \leq 1$,

$$
\|u(t)\|_{2} \leq C\left(t^{\epsilon(\alpha+1)-\alpha}|f(0)|_{2 \epsilon}+\left\|f^{\prime}(0)\right\|+\int_{0}^{t}\left\|f^{\prime \prime}\right\| d s\right),
$$

and

$\left\|u^{\prime}(t)\right\|_{2} \leq C\left(|f(0)|_{2}+t^{\epsilon(\alpha+1)-\alpha}\left|f^{\prime}(0)\right|_{2 \epsilon}+\left\|f^{\prime \prime}(0)\right\|+\int_{0}^{t}\left\|f^{\prime \prime \prime}\right\| d s\right)$, for $t \in(0, T]$. 
PROOF. The estimate for $\left\|u^{(m)}(t)\right\|$ follows at once from (5.9) using Theorem 5.5. To estimate $\|u(t)\|_{2}$, we write (5.1) as

$$
\omega_{\alpha} * A u=f-u^{\prime},
$$

and view this as an Abel integral equation for $A u$. By (5.15),

$$
A u=\left(\omega_{1} * A u\right)^{\prime}=\left[\omega_{1-\alpha} *\left(f-u^{\prime}\right)\right]^{\prime}=\omega_{1-\alpha} *\left(f^{\prime}-u^{\prime \prime}\right),
$$

where in the last step we used the fact that $f(0)-u^{\prime}(0)=\left(\omega_{\alpha} * A u\right)(0)=0$. Of course, (5.20) is just the well-known Abel inversion formula. Thus, using elliptic regularity, we have

$$
\|u(t)\|_{2} \leq C\|A u(t)\| \leq C \int_{0}^{t}(t-s)^{-\alpha}\left\|f^{\prime}(s)-u^{\prime \prime}(s)\right\| d s .
$$

We have seen already that

$$
\left\|u^{\prime \prime}(t)\right\| \leq C t^{\epsilon(\alpha+1)-1}|f(0)|_{2 \epsilon}+\left\|f^{\prime}(0)\right\|+\int_{0}^{t}\left\|f^{\prime \prime}\right\| d s,
$$

so the bound for $\|u(t)\|_{2}$ now follows using (5.15).

Next, we differentiate (5.20) to obtain

$$
A u^{\prime}=\omega_{1-\alpha} *\left(f^{\prime \prime}-u^{\prime \prime \prime}\right),
$$

noting that $f^{\prime}(0)-u^{\prime \prime}(0)=\left(\omega_{\alpha} * A u\right)^{\prime}(0)=0$ because $\left(\omega_{\alpha} * A u\right)^{\prime}=A u(0) \omega_{\alpha}+$ $\omega_{\alpha} * A u^{\prime}$ and $u(0)=v=0$. We know already that

$$
\left\|u^{\prime \prime \prime}(t)\right\| \leq C\left(t^{\alpha-1}|f(0)|_{2}+t^{\epsilon(\alpha+1)-1}\left|f^{\prime}(0)\right|_{2 \epsilon}+\left\|f^{\prime \prime}(0)\right\|+\int_{0}^{t}\left\|f^{\prime \prime \prime}\right\| d s\right),
$$

so the bound for $\left\|u^{\prime}(t)\right\|_{2}$ follows by a simple calculation.

Once again, consider as an example the regularity requirements for applying Theorem 3.1 with $r=2$, this time for the case of the singular kernel $\omega_{\alpha}, 0<$ $\alpha<1$, and using the piecewise-constant, product-integration rule (4.10), (4.11). The latter is first-order accurate by Lemma 4.7 , and we obtain the same error estimate (5.11) as before in the case of a smooth kernel. By Theorem 5.5,

$$
\left\|E^{\prime \prime}(t) v\right\| \leq C t^{\alpha-1}|v|_{2}, \quad\left\|E^{\prime}(t) v\right\|_{2} \leq C t^{\epsilon(\alpha+1)-1}|v|_{2+2 \epsilon},
$$


and combining these estimates with (5.21) and the last estimate of Theorem 5.6, we find that

$$
\begin{aligned}
\|v\|_{2}+\int_{0}^{T}\left(\left\|u_{t t}\right\|+\left\|u_{t}\right\|_{2}\right) d s & \\
& \leq C\left\{|v|_{2+2 \epsilon}+|f(0)|_{2}+\left|f^{\prime}(0)\right|_{2 \epsilon}+\left\|f^{\prime \prime}(0)\right\|+\int_{0}^{T}\left\|f^{\prime \prime \prime}(s)\right\| d s\right\}
\end{aligned}
$$

for $0<\epsilon \leq 1$. If $\epsilon<1 / 4$ then the boundary conditions which need to be satisfied for the right-hand side to be finite are that $v=f(0)=0$ on $\partial \Omega$, which may be compared with the more restrictive conditions (5.12) found earlier for the case when $\beta$ is smooth.

We close with a remark on the application of Theorem 3.2, concerning secondorder methods, in the case of the singular kernel $\omega_{\alpha}, 0<\alpha<1$, and using the piecewise-linear, product-integration rule (4.14). In view of Lemma 4.7, we have the error estimate

$$
\begin{aligned}
\left\|U^{n}-u\left(t_{n}\right)\right\| \leq C & h^{2}\left\{\|v\|_{2}+\int_{0}^{t_{n}}\left\|u_{t}\right\|_{2} d s\right\} \\
& +C k \int_{0}^{2 k}\left(\left\|u_{t t}\right\|+\left\|u_{t}\right\|_{2}\right) d s+C k^{2} \int_{k}^{t_{n}}\left(\left\|u_{t t t}\right\|+\left\|u_{t t}\right\|_{2}\right) d s,
\end{aligned}
$$

provided $v_{h}=R_{h} v$. For simplicity, we restrict ourselves to the homogenous equation, and obtain from Theorem 5.5, with $r=2(1+2 \alpha) /(1+\alpha)$,

$$
\left\|u_{t t}\right\|+\left\|u_{t}\right\|_{2} \leq C t^{\alpha-1}|v|_{r} \quad \text { and } \quad\left\|u_{t t t}\right\|+\left\|u_{t t}\right\|_{2} \leq C t^{\alpha-2}|v|_{r},
$$

which means that

$$
\left\|U^{n}-u\left(t_{n}\right)\right\| \leq C\left(h^{2}+k^{1+\alpha}\right)|v|_{r} .
$$

Thus, even though the regularity of the solution is not high enough to take full advantage of the second-order method, an improvement over a first-order method is manifest, more so the less singular the kernel.

\section{Numerical experiments}

In this section, we describe the results of some computations using the firstorder accurate, backward Euler method (3.2), and the second-order accurate 
scheme (3.7), together with appropriate quadrature rules. Some results for the Crank-Nicolson scheme (4.9) are also given. For simplicity, we deal with only one space dimension, choosing $\Omega=(0,1)$ and $A=-(d / d x)^{2}$. Thus, the eigenvalues and (normalised) eigenfunctions of $A$ are

$$
\lambda_{j}=(j \pi)^{2} \quad \text { and } \quad \varphi_{j}(x)=\sqrt{2} \sin (j \pi x) \quad \text { for } j \geq 1 .
$$

The discretisation in space is effected using piecewise-linear finite elements on a uniform mesh with $m$ subintervals, so that

$$
r=2, \quad h=1 / m,
$$

in the notation of Section 2. We remark that the Ritz projection $R_{h}$, defined by (2.3), is particularly simple in our case: $R_{h} u$ is just the piecewise-linear interpolant to $u$, i.e.,

$$
R_{h} u\left(x_{r}\right)=u\left(x_{r}\right), \quad \text { for } r=0, \ldots, m, \text { where } x_{r}=r h .
$$

This follows at once from the fact that

$$
A(u, \chi)=\int_{0}^{1} u^{\prime}(x) \chi^{\prime}(x) d x=\sum_{r=1}^{m}\left[u\left(x_{r}\right)-u\left(x_{r-1}\right)\right]\left[\chi\left(x_{r}\right)-\chi\left(x_{r-1}\right)\right],
$$

whenever $\chi$ is piecewise-linear.

The numerical methods were applied to the following two problems.

PROBLEM 1. Let $\beta$ be the smooth kernel

$$
\beta(t)=e^{-2 t}, \quad \text { for } t \geq 0 .
$$

Since $\beta^{\prime}=-2 \beta$, it is easy to see that the initial-value problem (5.3) is equivalent to

$$
\begin{gathered}
W_{\lambda}^{\prime \prime}+2 W_{\lambda}^{\prime}+\lambda W_{\lambda}=0, \quad \text { for } t>0, \\
W_{\lambda}(0)=1, \quad W_{\lambda}^{\prime}(0)=0,
\end{gathered}
$$

which has the solution

$$
W_{\lambda}(t)=e^{-t}\left[\cos (\mu t)+\mu^{-1} \sin (\mu t)\right],
$$

where $\mu=\sqrt{\lambda-1}$. As the initial data and inhomogeneous term, we choose simply

$$
v(x)=\sin (\pi x), \quad f(t, x)=\sin (2 \pi x) .
$$


By (5.4), the exact solution of (1.1) is

$$
\begin{aligned}
u(t, x)=e^{-t}[ & \left.\cos \left(\mu_{1} t\right)+\mu_{1}^{-1} \sin \left(\mu_{1} t\right)\right] \sin (\pi x) \\
& +\frac{1}{4 \pi^{2}}\left\{2-e^{-t}\left[2 \cos \left(\mu_{2} t\right)-\left(\mu_{2}-\mu_{2}^{-1}\right) \sin \left(\mu_{2} t\right)\right]\right\} \sin (2 \pi x),
\end{aligned}
$$

where $\mu_{1}=\sqrt{\pi^{2}-1}$ and $\mu_{2}=\sqrt{4 \pi^{2}-1}$.

PROBLEM 2. We choose the weakly-singular kernel (5.12) with $\alpha=1 / 2$, i.e.,

$$
\beta(t)=(\pi t)^{-1 / 2}, \quad \text { for } t>0 .
$$

By (5.16) and (5.17),

$$
W_{\lambda}(t)=\sum_{n=0}^{\infty}(-1)^{n} \frac{\left(\lambda t^{3 / 2}\right)^{n}}{\Gamma\left[1+\frac{3}{2} n\right]}, \quad t \geq 0,
$$

so, with $v(x)=\sin (\pi x)$ and $f(t, x)=\sin (\pi x)$, the exact solution is

$$
u(t, x)=\left\{\sum_{n=0}^{\infty}(-1)^{n} \frac{\left(\pi^{2} t^{3 / 2}\right)^{n}}{\Gamma\left[1+\frac{3}{2} n\right]}+t \sum_{n=0}^{\infty}(-1)^{n} \frac{\left(\pi^{2} t^{3 / 2}\right)^{n}}{\Gamma\left[2+\frac{3}{2} n\right]}\right\} \sin (\pi x) .
$$

We used this series representation when computing the errors in our numerical solutions. Roundoff was not a serious problem because we only considered $0 \leq t \leq 2$. (For large $t$, the series in (6.3) are subject to catastrophic cancellation, in much the same way as occurs with the Taylor series of $e^{-t}$.)

Some computed values of the error $\left\|U^{n}-u\left(t_{n}\right)\right\|$ are set out in Tables 1-8, with the numbers written in Fortran E-format. We used a composite fourpoint Gauss-Legendre rule to evaluate the $L_{2}$-norms, an approximation which is accurate to $O\left(h^{4}\right)$ if $u$ is a smooth function of $x$. The same quadrature formula was used to evaluate the $L_{2}$ inner products $\left(f_{n}, \chi\right)$, in (3.2) and (3.7), and $\left(f_{n-1 / 2}, \chi\right)$, in (4.9), with $\chi$ a piecewise-linear basis function.

For the approximate initial data $v_{h}$, we chose in every case the piecewiselinear interpolant to the exact initial data $v$. In view of our earlier remarks concerning the Ritz projection, this meant that

$$
v_{h}=R_{h} v
$$




\begin{tabular}{r|cccccc|}
\hline & $m=4$ & 8 & 16 & 32 & 64 & 128 \\
\hline$t=0.0$ & $.216 \mathrm{E}+0$ & $.557 \mathrm{E}-1$ & $.140 \mathrm{E}-1$ & $.352 \mathrm{E}-2$ & $.880 \mathrm{E}-3$ & $.220 \mathrm{E}-3$ \\
0.5 & $.717 \mathrm{E}-1$ & $.393 \mathrm{E}-1$ & $.287 \mathrm{E}-1$ & $.182 \mathrm{E}-1$ & $.103 \mathrm{E}-1$ & $.551 \mathrm{E}-2$ \\
1.0 & $.245 \mathrm{E}+0$ & $.161 \mathrm{E}+0$ & $.934 \mathrm{E}-1$ & $.503 \mathrm{E}-1$ & $.261 \mathrm{E}-1$ & $.133 \mathrm{E}-1$ \\
1.5 & $.737 \mathrm{E}-1$ & $.585 \mathrm{E}-1$ & $.414 \mathrm{E}-1$ & $.256 \mathrm{E}-1$ & $.145 \mathrm{E}-1$ & $.777 \mathrm{E}-2$ \\
2.0 & $.124 \mathrm{E}+0$ & $.876 \mathrm{E}-1$ & $.553 \mathrm{E}-1$ & $.315 \mathrm{E}-1$ & $.169 \mathrm{E}-1$ & $.879 \mathrm{E}-2$ \\
\hline
\end{tabular}

TABLE 1: Backward Euler method; Problem $1 ; k=h$.

\begin{tabular}{r|cccccc|}
\hline & $m=4$ & 8 & 16 & 32 & 64 & 128 \\
\hline$t=0.0$ & $.216 \mathrm{E}+0$ & $.557 \mathrm{E}-1$ & $.140 \mathrm{E}-1$ & $.352 \mathrm{E}-2$ & $.880 \mathrm{E}-3$ & $.220 \mathrm{E}-3$ \\
0.5 & $.271 \mathrm{E}+0$ & $.173 \mathrm{E}+0$ & $.104 \mathrm{E}+0$ & $.590 \mathrm{E}-1$ & $.319 \mathrm{E}-1$ & $.167 \mathrm{E}-1$ \\
1.0 & $.636 \mathrm{E}-1$ & $.248 \mathrm{E}-1$ & $.779 \mathrm{E}-2$ & $.171 \mathrm{E}-2$ & $.541 \mathrm{E}-4$ & $.280 \mathrm{E}-3$ \\
1.5 & $.551 \mathrm{E}-1$ & $.467 \mathrm{E}-1$ & $.314 \mathrm{E}-1$ & $.185 \mathrm{E}-1$ & $.101 \mathrm{E}-1$ & $.529 \mathrm{E}-2$ \\
2.0 & $.612 \mathrm{E}-2$ & $.350 \mathrm{E}-2$ & $.550 \mathrm{E}-2$ & $.472 \mathrm{E}-2$ & $.310 \mathrm{E}-2$ & $.179 \mathrm{E}-2$ \\
\hline
\end{tabular}

TABLE 2: Backward Euler method; Problem $2 ; k=h$.

Tables 1-4 list some results for the backward Euler method (3.2) applied to Problems 1 and 2. For Problem 1, the integration rule $q_{n}$ was the right-hand rectangle rule (4.5), whereas for Problem 2, the right-hand, piecewise-constant, product-integration rule (4.10) was used, in order to handle the singular kernel (6.2). From the error estimate (5.11) and the discussion following Theorem 5.6, we see that for both problems,

$$
\left\|U^{n}-u\left(t_{n}\right)\right\|=O\left(h^{2}+k\right) .
$$

The results in Tables 1 and 2 arose from taking $k=h$, and as expected the errors behave as $O\left(\mathrm{~m}^{-1}\right)$, except at $t=0$ where we see simply the interpolation error $\left\|v_{h}-v\right\|=O\left(m^{-2}\right)$. In some cases, the error does not enter the asymptotic regime until $m$ is quite large, and there are irregularities in Table 2 because of sign changes that occur in the pointwise errors around $t=1$ and $t=2$. Choosing the much smaller time step $k=h^{2}$ reduces the error to $O\left(m^{-2}\right)$ for all $t_{n}$, as confirmed in Tables 3 and 4.

\begin{tabular}{|r|ccccc|}
\hline & $m=2$ & 4 & 8 & 16 & 32 \\
\hline$t=0.0$ & $.763 \mathrm{E}+0$ & $.216 \mathrm{E}+0$ & $.557 \mathrm{E}-1$ & $.140 \mathrm{E}-1$ & $.352 \mathrm{E}-2$ \\
0.5 & $.174 \mathrm{E}+0$ & $.817 \mathrm{E}-1$ & $.236 \mathrm{E}-1$ & $.612 \mathrm{E}-2$ & $.153 \mathrm{E}-2$ \\
1.0 & $.248 \mathrm{E}+0$ & $.864 \mathrm{E}-1$ & $.256 \mathrm{E}-1$ & $.690 \mathrm{E}-2$ & $.177 \mathrm{E}-2$ \\
1.5 & $.972 \mathrm{E}-1$ & $.570 \mathrm{E}-1$ & $.208 \mathrm{E}-1$ & $.593 \mathrm{E}-2$ & $.153 \mathrm{E}-2$ \\
2.0 & $.131 \mathrm{E}+0$ & $.669 \mathrm{E}-1$ & $.202 \mathrm{E}-1$ & $.541 \mathrm{E}-2$ & $.136 \mathrm{E}-2$ \\
\hline
\end{tabular}

TABLE 3: Backward Euler method; Problem $1 ; k=h^{2}$. 


\begin{tabular}{|r|ccccc|}
\hline & $m=2$ & 4 & 8 & 16 & 32 \\
\hline$t=0.0$ & $.763 \mathrm{E}+0$ & $.216 \mathrm{E}+0$ & $.557 \mathrm{E}-1$ & $.140 \mathrm{E}-1$ & $.352 \mathrm{E}-2$ \\
0.5 & $.271 \mathrm{E}+0$ & $.946 \mathrm{E}-1$ & $.270 \mathrm{E}-1$ & $.716 \mathrm{E}-2$ & $.184 \mathrm{E}-2$ \\
1.0 & $.700 \mathrm{E}-1$ & $.171 \mathrm{E}-1$ & $.401 \mathrm{E}-2$ & $.957 \mathrm{E}-3$ & $.236 \mathrm{E}-3$ \\
1.5 & $.484 \mathrm{E}-1$ & $.269 \mathrm{E}-1$ & $.883 \mathrm{E}-2$ & $.238 \mathrm{E}-2$ & $.600 \mathrm{E}-3$ \\
2.0 & $.229 \mathrm{E}-1$ & $.102 \mathrm{E}-1$ & $.374 \mathrm{E}-2$ & $.108 \mathrm{E}-2$ & $.287 \mathrm{E}-3$ \\
\hline
\end{tabular}

TABLE 4: Backward Euler method; Problem 2; $k=h^{2}$.

In Table 5 are listed the errors when the second-order, backward differencing scheme (3.7) was used to solve Problem 1 . In this case, $q_{n}$ was the trapezoidal rule (4.7), which means, in view of (6.4) and the remark following Lemma 4.5, that

$$
\left\|U^{n}-u\left(t_{n}\right)\right\|=O\left(h^{2}+k^{2}\right),
$$

so even with the time step $k=h$ the error is $O\left(m^{-2}\right)$. For comparison, we also solved Problem 1 using the Crank-Nicolson scheme (4.9), (4.10) with the same time step $k=h$. The results are shown in Table 6, and indicate that the errors are $O\left(m^{-2}\right)$.

\begin{tabular}{|r|ccccc|}
\hline & $m=4$ & 8 & 16 & 32 & 64 \\
\hline$t=0.0$ & $.216 \mathrm{E}+0$ & $.557 \mathrm{E}-1$ & $.140 \mathrm{E}-1$ & $.352 \mathrm{E}-2$ & $.880 \mathrm{E}-3$ \\
0.5 & $.117 \mathrm{E}+0$ & $.372 \mathrm{E}-1$ & $.106 \mathrm{E}-1$ & $.284 \mathrm{E}-2$ & $.731 \mathrm{E}-3$ \\
1.0 & $.866 \mathrm{E}-1$ & $.372 \mathrm{E}-1$ & $.117 \mathrm{E}-1$ & $.310 \mathrm{E}-2$ & $.779 \mathrm{E}-3$ \\
1.5 & $.567 \mathrm{E}-1$ & $.265 \mathrm{E}-1$ & $.100 \mathrm{E}-1$ & $.283 \mathrm{E}-2$ & $.729 \mathrm{E}-3$ \\
2.0 & $.720 \mathrm{E}-1$ & $.194 \mathrm{E}-1$ & $.622 \mathrm{E}-2$ & $.174 \mathrm{E}-2$ & $.443 \mathrm{E}-3$ \\
\hline
\end{tabular}

TABLE 5: Second order scheme; Problem $1 ; k=h$.

\begin{tabular}{|r|ccccc|}
\hline & $m=4$ & 8 & 16 & 32 & 64 \\
\hline$t=0.0$ & $.216 \mathrm{E}+0$ & $.557 \mathrm{E}-1$ & $.140 \mathrm{E}-1$ & $.352 \mathrm{E}-2$ & $.880 \mathrm{E}-3$ \\
0.5 & $.118 \mathrm{E}+0$ & $.302 \mathrm{E}-1$ & $.763 \mathrm{E}-2$ & $.192 \mathrm{E}-2$ & $.482 \mathrm{E}-3$ \\
1.0 & $.793 \mathrm{E}-1$ & $.204 \mathrm{E}-1$ & $.505 \mathrm{E}-2$ & $.125 \mathrm{E}-2$ & $.312 \mathrm{E}-3$ \\
1.5 & $.852 \mathrm{E}-1$ & $.222 \mathrm{E}-1$ & $.555 \mathrm{E}-2$ & $.139 \mathrm{E}-2$ & $.348 \mathrm{E}-3$ \\
2.0 & $.319 \mathrm{E}-1$ & $.933 \mathrm{E}-2$ & $.246 \mathrm{E}-2$ & $.625 \mathrm{E}-3$ & $.157 \mathrm{E}-3$ \\
\hline
\end{tabular}

TABle 6: Crank-Nicolson scheme; Problem $1 ; k=h$.

Finally, Problem 2 was solved using the second-order scheme (3.7), and the resulting errors are given in Tables 7 and 8 . We used the piecewise-linear, 
product-integration rule (4.14), so that by Theorem 3.2 and Lemma 4.8,

$$
\left\|U^{n}-u\left(t_{n}\right)\right\|=O\left(h^{2}+k^{3 / 2}\right) ;
$$

cf. the concluding remarks to Section 5. We first chose $k=h$, and as shown in Table 7 the errors were consistent with the projected $O\left(m^{-3 / 2}\right)$ behaviour. After this, we chose $k$ such that $1 / k$ was the smallest even number for which $k^{3 / 2} \leq h^{2}$, and Table 8 accordingly shows errors of order $O\left(m^{-2}\right)$.

\begin{tabular}{|r|ccccc|}
\hline & $m=4$ & 8 & 16 & 32 & 64 \\
\hline$t=0.0$ & $.216 \mathrm{E}+0$ & $.557 \mathrm{E}-1$ & $.140 \mathrm{E}-1$ & $.352 \mathrm{E}-2$ & $.880 \mathrm{E}-3$ \\
0.5 & $.420 \mathrm{E}-1$ & $.333 \mathrm{E}-1$ & $.165 \mathrm{E}-1$ & $.592 \mathrm{E}-2$ & $.198 \mathrm{E}-2$ \\
1.0 & $.497 \mathrm{E}-1$ & $.118 \mathrm{E}-1$ & $.250 \mathrm{E}-2$ & $.256 \mathrm{E}-3$ & $.167 \mathrm{E}-3$ \\
1.5 & $.143 \mathrm{E}-1$ & $.783 \mathrm{E}-2$ & $.255 \mathrm{E}-2$ & $.622 \mathrm{E}-3$ & $.132 \mathrm{E}-3$ \\
2.0 & $.340 \mathrm{E}-1$ & $.751 \mathrm{E}-2$ & $.161 \mathrm{E}-2$ & $.419 \mathrm{E}-3$ & $.127 \mathrm{E}-3$ \\
\hline
\end{tabular}

TABLE 7: Second order scheme; Problem $2 ; k=h$.

\begin{tabular}{|r|ccccc|}
\hline & $m=4$ & 8 & 16 & 32 & 64 \\
\hline$t=0.0$ & $.216 \mathrm{E}+0$ & $.557 \mathrm{E}-1$ & $.140 \mathrm{E}-1$ & $.352 \mathrm{E}-2$ & $.880 \mathrm{E}-3$ \\
0.5 & $.212 \mathrm{E}-1$ & $.126 \mathrm{E}-1$ & $.267 \mathrm{E}-2$ & $.626 \mathrm{E}-3$ & $.140 \mathrm{E}-3$ \\
1.0 & $.134 \mathrm{E}-1$ & $.336 \mathrm{E}-2$ & $.109 \mathrm{E}-2$ & $.369 \mathrm{E}-3$ & $.119 \mathrm{E}-3$ \\
1.5 & $.191 \mathrm{E}-1$ & $.507 \mathrm{E}-2$ & $.108 \mathrm{E}-2$ & $.249 \mathrm{E}-3$ & $.633 \mathrm{E}-4$ \\
2.0 & $.928 \mathrm{E}-2$ & $.222 \mathrm{E}-2$ & $.475 \mathrm{E}-3$ & $.118 \mathrm{E}-3$ & $.316 \mathrm{E}-4$ \\
\hline
\end{tabular}

TABLE 8: Second order scheme; Problem $2 ; k^{3 / 2} \approx h^{2}$.

\section{References}

[1] P. L. Butzer and R. J. Nessel, Fourier Analysis and Approximation (Birkhäuser Verlag, 1971).

[2] U. Jin Choi and R. C. MacCamy, "Fractional order Volterra equations", in Volterra Integrodifferential Equations in Banach Spaces and Applications in Pitman Research Notes in Mathematics, Volume 190 (eds. G. Da Prato and M. Iannelli), (Longman, Harlow, UK, and Wiley, New York, 1989) 231-245.

[3] P. G. Ciarlet, The Finite Element Method for Elliptic Problems (North-Holland, Amsterdam, 1978). 
[4] M. Crouzeix and V. Thomee, "The stability in $L_{p}$ and $W_{p}^{1}$ of the $L_{2}$ projection onto finite element function spaces", Math. Comp. 48 (1987) 521-532.

[5] C. M. Dafermos and J. A. Nohel, "Energy methods for nonlinear hyperbolic Volterra integro-differential equations", Comm. Partial Differential Equations 4 (1979) 219-278.

[6] J. Douglas and T. Dupont, "Galerkin methods for parabolic equations", SIAM J. Num. Anal. 7 (1970) 575-526.

[7] G. Fairweather, "Spline collocation methods for a class of partial integro-differential equations", preprint (1989).

[8] P. Grisvard, "Caractérisation de quelques espaces d'interpolation", Arch. Rational Mech. Anal. 25 (1967) 40-63.

[9] J. L. Lions and E. Magenes, Non-Homogeneous Boundary Value Problems and Applications I (Springer Verlag, Berlin, Heidelberg, New York, 1972).

[10] S.-O. Londen, "On an integrodifferential Volterra equation with a maximal monotone mapping”, J. Differential Equations 27 (1978) 405-420.

[11] J.-C. López-Marcos, "A difference scheme for a nonlinear partial integrodifferential equation", SIAM J. Numer. Anal. 27 (1990) 20-31.

[12] R. C. MacCamy, "An integro-differential equation with application to heat flow", Quart. Appl. Math. 35 (1977) 1-19.

[13] R. C. MacCamy, "A model for one-dimensional nonlinear viscoelasticity", Quart. Appl. Math. 35 (1977) 21-33.

[14] R. C. MacCamy and J. S. W. Wong, "Stability theorems for some functional equations", Trans. Amer. Math. Soc. 164 (1972) 1-37.

[15] B. Neta, "Numerical solution of a nonlinear integro-differential equation", J. Math. Anal. Appl 98 (1982) 598-611.

[16] J. M. Sanz-Serna, "A numerical method for a partial integro-differential equation", SIAM J. Numer. Anal. 25 (1988) 319-327.

[17] I. H. Sloan and V. Thomee, "Time discretization of an integro-differential equation of parabolic type", SIAM J. Numer. Anal. 23 (1986) 1052-1061.

[18] O. J. Staffans, "On a nonlinear hyperbolic Volterra equation", SIAM J. Math. Anal. 11 (1980) 793-812.

[19] V. Thomée, "Galerkin finite element methods for parabolic problems", in Lecture Notes in Mathematics 1054, (Springer-Verlag, Berlin, 1984).

[20] Y. Yan, "Fast inverse Laplace transform for some time dependent problems with memory", preprint (1990).

[21] Y. Yan and G. Fairweather, "Orthogonal spline collocation methods for some partial integrodifferential equations", preprint (1990).

[22] A. Zygmund, Trigonometric series I (Cambridge University Press, Cambridge, 1959). 